\title{
WHAT THE FUTURE HOLDS FOR REGENERATIVE ENDODONTICS: NOVEL ANTIMICROBIALS AND REGENERATIVE STRATEGIES
}

\author{
M. Matoug-Elwerfelli ${ }^{1, *}$, H. Nazzal ${ }^{2}$, M. Duggal ${ }^{3}$ and R. El-Gendy $y^{4,5}$ \\ ${ }^{1}$ Department of Clinical Dental Science, Princess Nourah Bint Abdulrahman University, Riyadh, KSA \\ ${ }^{2}$ Hamad Dental Centre, Hamad Medical Corporation, Doha, Qatar \\ ${ }^{3}$ College of Dental Medicine, Qatar University Health, Qatar University, Doha, Qatar \\ ${ }^{4}$ Division of Oral Biology, School of Dentistry, University of Leeds, UK \\ ${ }^{5}$ Department of Oral Pathology, Faculty of Dentistry, Suez Canal University, Ismailia, Egypt
}

\begin{abstract}
Regenerative/revitalisation endodontic techniques are increasingly used as a treatment approach for the management of immature permanent teeth with necrotic pulps. Different chemical irrigants and medicaments are routinely used clinically for intra-canal disinfection. However, despite remarkable progress in this field, coronal discolouration, cell cytotoxicity, difficulty of removal of organic biofilm from the root canal, development of sensitisation and antimicrobial resistance are still challenges to this line of treatment. This review critically discusses and challenges the current status quo of antimicrobials used in regenerative endodontics and sheds the light on future alternative antimicrobial materials with regenerative potential.
\end{abstract}

Keywords: Antimicrobials, antibiotics, disinfection, biomaterials, regenerative endodontics, drug delivery, dental pulp stem cells, pulp regeneration.

*Address for correspondence: Dr Manal Matoug-Elwerfelli, Department of Clinical Dental Science, Princess Nourah Bint Abdulrahman University, Riyadh, Kingdom of Saudi Arabia.

Telephone number: +966 553713047 Email: mmelwerfelli@pnu.edu.sa

Copyright policy: This article is distributed in accordance with Creative Commons Attribution Licence (http://creativecommons.org/licenses/by-sa/4.0/).

\begin{tabular}{|c|c|c|c|}
\hline & List of Abbreviations & $\begin{array}{l}\text { MIC } \\
\text { MTA }\end{array}$ & $\begin{array}{l}\text { minimum inhibitory concentrations } \\
\text { mineral trioxide aggregate }\end{array}$ \\
\hline A. naeslundii & Actinomyces naeslundii & $\mathrm{NaOCl}$ & sodium hypochlorite \\
\hline A. radicidentis & Actinomyces radicidentis & NFkB & nuclear factor kappa B \\
\hline Ag-GO & $\begin{array}{l}\text { AgNPs synthesised on an aqueous } \\
\text { graphene oxide matrix }\end{array}$ & $\begin{array}{l}\text { P. acnes } \\
\text { PBS }\end{array}$ & $\begin{array}{l}\text { Propionibacterium acnes } \\
\text { phosphate-buffered saline }\end{array}$ \\
\hline AgNPs & silver nanoparticles & pERK & protein R-like endoplasmic reticulum \\
\hline C. albicans & Candida albicans & & kinase \\
\hline C. longa & Curcuma longa & PDT & photodynamic therapy \\
\hline $\mathrm{Ca}(\mathrm{OH})_{2}$ & calcium hydroxide & POVI & povidone iodine \\
\hline CFUs & colony forming units & PPM & part per million \\
\hline $\mathrm{CHX}$ & chlorhexidine gluconate & qPCR & quantitative real-time polymerase \\
\hline CLSM & confocal laser scanning microscope & & chain reaction \\
\hline DAP & double antibiotic paste & RET & regenerative/revitalisation endodontic \\
\hline DMSO & dimethyl sulfphoxide & & techniques \\
\hline DPSCs & dental pulp stem cells & rGO-Cur & reduced graphene oxide-curcumin \\
\hline E. coli & Escherichia coli & S. aureus & Staphylococcus aureus \\
\hline E. faecalis & Enterococcus faecalis & S. enterica & Salmonella enterica \\
\hline EDTA & ethylenediaminetetraacetic acid & S. epidermidis & Staphylococcus epidermidis \\
\hline EEP & ethanol extract of propolis & S. mitis & Streptococcus mitis \\
\hline ERK & extracellular signal-regulated kinases & S. mutans & Streptococcus mutans \\
\hline FtsZ & Filamenting temperature-sensitive & $\begin{array}{l}\text { SCAP } \\
\text { SEM }\end{array}$ & $\begin{array}{l}\text { stem cells of the apical papilla } \\
\text { scanning electron microscope }\end{array}$ \\
\hline L. monocytogenes & $\begin{array}{l}\text { mutant } Z \\
\text { Listeria monocytogenes }\end{array}$ & TAMP & tailored amorphous multiporous \\
\hline LED & light emitting diode & TAP & triple antibiotic paste \\
\hline MBC & minimum bactericidal concentration & TGF $\beta-1$ & transforming growth factor $\beta 1$ \\
\hline MCJ & Morinda citrifolia juice & TS & tryptone soy \\
\hline
\end{tabular}




\section{Introduction}

RET are becoming widely used in the management of immature permanent teeth with necrotic pulp tissues, in which other alternative treatment options offer less successful outcomes (Galler, 2016). RET are biologically based treatments that aim to replace damaged tissue structures, including the pulpdentine complex (Murray et al., 2007). This treatment strategy, if successful, could potentially allow the continuation of root maturation, therefore, improving the long-term prognosis of these compromised teeth (Conde et al., 2016; Garcia-Godoy and Murray, 2012). Clinically this treatment involves no or very minimal root canal instrumentation, disinfection, followed by induction of apical bleeding into the root canal space (Banchs and Trope, 2004; Iwaya et al., 2001). Due to the limited or no mechanical instrumentation, microbial elimination is mainly dependent on antimicrobial agents for sufficient canal disinfection. Despite remarkable progress in the field of RET over the past decade, several challenges remain (Tong et al., 2017), such as the questionable efficacy of the currently available disinfection techniques in promoting continuation of root development (Fouad, 2020).

Furthermore, RET is a stem cell-based regeneration strategy. Hence the balance between root canal disinfection and keeping the microenvironment as hospitable as possible for the stem cells to regenerate the pulp-dentine complex is of prime importance (Kim et al., 2018). Many of the currently used intra-canal irrigants such as $\mathrm{NaOCl}$ and $\mathrm{CHX}$ were proven to have a cytotoxic effect on stem cells (Martin et al., 2014; Widbiller et al., 2019). The use of topical antibiotic pastes are effective endodontic antimicrobials; however, in addition to tooth discoloration caused by some of the antibiotic combinations (Lenherr et al., 2012), a level of cytotoxicity on stem cells in a concentrationdependent manner is of clinical concern (Ruparel et al., 2012). Although using topical antibiotic pastes at lower concentrations provided adequate canal disinfection, such mixtures are difficult to prepare and obtain. The argument about their contribution to the global challenge of antimicrobial resistance at such low concentrations is yet to be settled (Ayoub et al., 2020; Yadlapati et al., 2017; Yadlapati et al., 2014).

Therefore, the development of alternative disinfectant strategies with sufficient antimicrobial efficacy, biocompatibility, and regenerative potential (with the least harm to the surrounding conductive microenvironment) is of importance in this field. The achievement of a safer and more predictable regenerative outcome is clinically required (Diogenes et al., 2014; Fouad, 2020). With the current advances in tissue engineering, this gap is being addressed through several innovative natural and synthetic strategies, such as alternative antimicrobial agents, nanobased delivery systems, and novel photosensitisers combined with PDT which initiate tissue regeneration and possess an innate antimicrobial activity (Chung and Park, 2017; Samiei et al., 2016). A schematic illustration depicting limitations of currently used antimicrobial strategies and the potential benefits of alternative RET antimicrobial strategies is provided (Fig. 1).

Taking all of the above into consideration, it seems comprehensible to develop an alternative antimicrobial able to disinfect the root canal system without compromising the regenerative environment. The aim of this review is to critically discuss and challenge the current status quo of antimicrobials

\section{Current versus alternative antimicrobial stratergies for regenerative endodontics}

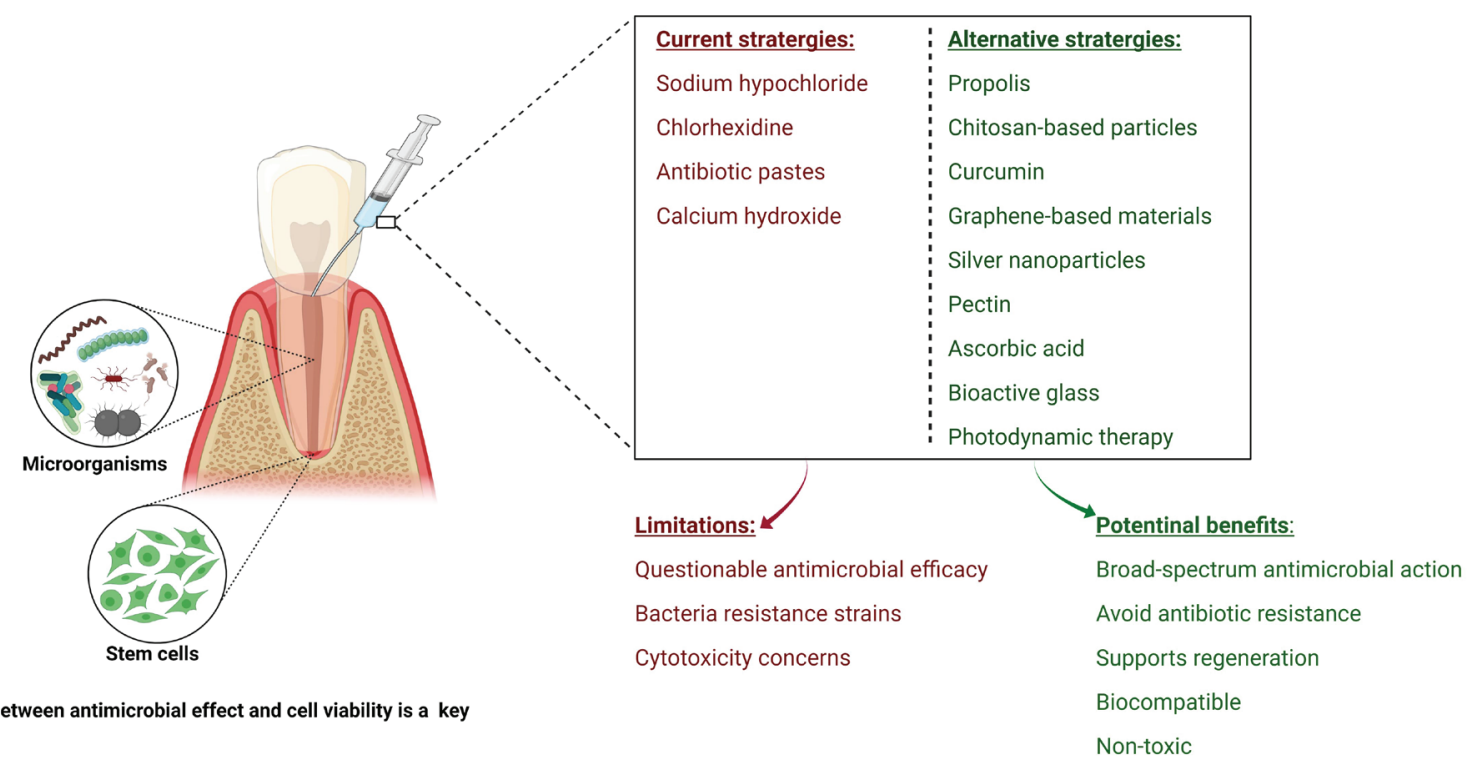

Fig. 1. Schematic illustration depicting limitations of currently used antimicrobial strategies and the potential benefits of alternative RET antimicrobial strategies. Created with BioRender.com. 
used in regenerative endodontics, and to shed the light on future alternative antimicrobial materials with regenerative potential.

\section{Literature search and scope of the review}

An electronic search of PubMed and Elsevier's Scopus was undertaken with appropriate $\mathrm{MeSH}$ terms and various keyword combinations including "antimicrobial", "antibiotic", "disinfection", "dentistry", "pulp revascularisation", "pulp regeneration", "regenerative endodontic", "drug delivery", "biomaterials", and "dental pulp stem cells". The reference list of the relevant articles resulting from database searches was further handscreened. No limits were applied to the year of publication and only English language literature was included. However, due to the scope and extent of this search, a wide-ranging comprehensive narrative review of antimicrobial strategies in regenerative endodontics rather than a systematic review was undertaken.

\section{Current antimicrobial strategies used in RET}

A critical summary of the most commonly used disinfectant agents (irrigants and medicaments) in terms of their antimicrobial efficacy and biocompatibility will be discussed below as these have been extensively reviewed in the literature (Diogenes et al., 2014; Kim et al., 2018; Martin et al., 2014).

\section{Intra-canal irrigation}

$\mathrm{NaOCl}$ is one of the oldest endodontic irrigants and reported in most published RET studies, albeit in various concentrations ranging from $1-6 \%$ (Tong et al., 2020). $\mathrm{NaOCl}$ solution is regarded as the irrigant of choice mainly due to its bacteriostatic, bactericidal, and tissue dissolution properties (Bryce et al., 2009; Zehnder, 2006). The ability of $\mathrm{NaOCl}$ to dissolve organic tissue is well-documented, as well as its negative effects on the mechanical properties of root dentine (Dotto et al., 2020; Pascon et al., 2009). These mechanical alterations are most likely due to the proteolytic action of concentrated hypochlorite solution on the collagen dentine matrix (Zehnder, 2006). Alterations in the dentine-matrix composition including the denaturation of growth factors and attachment proteins are likely to affect the fate of stem cells (Diogenes et al., 2014). Furthermore, $\mathrm{NaOCl}$ at a concentration between 5-6\%, has shown detrimental effects on stem cell numbers and survival as well as loss of odontoblast-like phenotype differentiation both in vitro and in vivo (Casagrande et al., 2010; Galler et al., 2011).

From a biological perspective, a concentrationdependent effect of $\mathrm{NaOCl}$ on the survival of SCAP has been shown, with $6 \% \mathrm{NaOCl}$ showing the greatest reduction in stem cell survival. This resulted in the recommendation for using a low $\mathrm{NaOCl}$ concentration of $1.5 \%$ (Web ref.1; Martin et al., 2014; Trevino et al., 2011). However, controversies remain in terms of the ability of low $\mathrm{NaOCl}$ concentrations to completely eradicate infected biofilms (Ma et al., 2015; Tagelsir et al., 2016). In an attempt to reduce such effect, $17 \%$ EDTA has been recommended following the use of $\mathrm{NaOCl}$ irrigation. This step has been shown to reduce $\mathrm{NaOCl}$ detrimental side effects on stem cell survival, regardless of the $\mathrm{NaOCl}$ concentration used (Martin et al., 2014; Trevino et al., 2011). Furthermore, combining EDTA within a given irrigation protocol was also found to significantly increase the release of growth factors such as TGF $\beta-1$ into the root canal space, hence inducing DPSCs migration and differentiation to odontoblasts (Zeng et al., 2016).

CHX is also one of the well known endodontic disinfectant agents used in RET and clinically available in the form of an aqueous solution or gel preparation with a dilution range of $0.12 \%$ to $2 \%$ (Okino et al., 2004). However, the use of this irrigant is less prevalent within RET protocols as highlighted in a recent survey, in which only $11.4 \%$ of respondents used $\mathrm{CHX}$ as the sole disinfectant, while $22.2 \%$ reported a combined use of $\mathrm{CHX}$ and $\mathrm{NaOCl}$ (Tong et al., 2020). CHX benefits from broadspectrum antimicrobial and intra-canal substantivity (residual effect) properties (Martin et al., 2014; Okino et al., 2004; Trevino et al., 2011). The use of $2 \%$ CHX has also been shown to cause unfavourable effects on stem cell survival (Trevino et al., 2011) and attachment (Ring et al., 2008), with direct cytotoxicity effect in a concentration-dependent manner (Widbiller et al., 2019). On the contrary, CHX lacks organic tissue dissolution ability (Okino et al., 2004); therefore, its effect on biofilm disruption is questioned (Bryce et al., 2009; Trevino et al., 2011).

\section{Intra-canal medicaments}

Intra-canal medicaments are commonly used interappointment antimicrobial RET dressing (Tong et al., 2020) and broadly divided into two groups; topical antibiotic pastes or $\mathrm{Ca}(\mathrm{OH})_{2}$. Topical antibiotic pastes used within RET mainly include; TAP (ciprofloxacin, metronidazole, and minocycline), DAP (ciprofloxacin and metronidazole), and other modified formulations (Tong et al., 2020). Although sufficient antimicrobial efficacy is one of the main advantages behind the use of topical antibiotic pastes, clinical limitations have been raised (Ribeiro et al., 2020).

Complete removal of the applied antibiotic paste is crucially important for a successful regenerative outcome and to avoid unwanted, possibly longterm, side effects. Unfortunately, studies have demonstrated that a significant amount of antibiotic pastes ( $88 \%$ residual) remains within the root canal system following current irrigation techniques (Berkhoff et al., 2014). Indirect negative effects, such as reduction in dentinal strength and fracture resistance, are reported as early as 1 week post- 
application. These effects were mainly linked to the strong demineralisation effect and the acidic nature of antibiotic pastes on the surrounding dentine matrix (Yassen et al., 2013a; Yassen et al., 2013b). These structural changes are thought to affect the fate of stem cells and hinder their regenerative potential, consequently resulting in inconsistent clinical results related to continuation of root maturation, thickening of root dentine, and apical closure (Tong et al., 2017).

A concentration-dependent detrimental effect of various topical antibiotic pastes on human SCAP survival has been shown in vitro (Althumairy et al., 2014; Ruparel et al., 2012). Therefore, to achieve optimal results, various antibiotic mixtures and concentrations have been tested. A low TAP and DAP concentration of $1 \mathrm{mg} / \mathrm{mL}$ provided sufficient antimicrobial efficacy with no reported negative effects on the viability of SCAP when compared to higher concentrations $(1,000 \mathrm{mg} / \mathrm{mL})$ (Althumairy et al., 2014). Furthermore, the potential development of bacterial resistance biofilms and/or sensitisation has been raised (Berkhoff et al., 2014; Stewart and William Costerton, 2001). Coronal discolouration is also a commonly reported side effect of the TAP use, which is largely linked to minocycline, a semisynthetic tetracycline antibiotic (Kim et al., 2010; Lenherr et al., 2012; Sato et al., 1996). However, despite omitting minocycline within RET protocols tooth discoloration continued to be reported (Tong et al., 2017).

$\mathrm{Ca}(\mathrm{OH})_{2}$ is another widely used intracanal medicament advocated to overcome the undesirable effects of the topical antibiotic pastes and recommended by the European Society of Endodontology for short-term clinical application (Galler et al., 2016). Material advantages such as availability and ease of removal from the root canal are documented (Berkhoff et al., 2014; Nazzal et al., 2018).

Nevertheless, conflicting antimicrobial efficacy of $\mathrm{Ca}(\mathrm{OH})_{2}$, as an intra-canal dressing, has been reported (Ribeiro et al., 2020). The ability of $\mathrm{Ca}(\mathrm{OH})_{2}$ to eradicate specific bacteria, such as E. faecalis, and yeasts from the root canal systems has been questioned (Krithikadatta et al., 2007; Zehnder et al., 2004). More recently, a clinical molecular-based study showed comparable antimicrobial efficacy and regenerative outcome following the use of TAP and a combined $\mathrm{Ca}(\mathrm{OH})_{2} / \mathrm{CHX}$ paste (de-Jesus-Soares et al., 2020). From a biological perspective, $\mathrm{Ca}(\mathrm{OH})_{2}$ provided an environment conducive to stem cell survival and proliferation (Althumairy et al., 2014; Ruparel et al., 2012). However, the possible side effects of $\mathrm{Ca}(\mathrm{OH})_{2}$ on the biological property of dentinematrix-derived growth factors have been highlighted and requires consideration (Kim et al., 2018).

\section{Possible alternative antimicrobial strategies with a regenerative potential for RET}

The development and characterisation of the next generation novel materials that can enhance the regeneration of pulp-dentine complex as well as provide sufficient antimicrobial properties is a recent focus area for a safer and predictable regenerative outcome (Chung and Park, 2017). Indeed, a recent scoping review of the literature concluded that trends towards alternative antimicrobials are promising and deserve future consideration (Ribeiro et al., 2020). Word cloud highlighting of both natural and synthetic alternative antimicrobial materials and strategies for potential use in RET was performed. The more a substance has been researched, within this specific field, the larger it appears in the word cloud (Fig. 2).

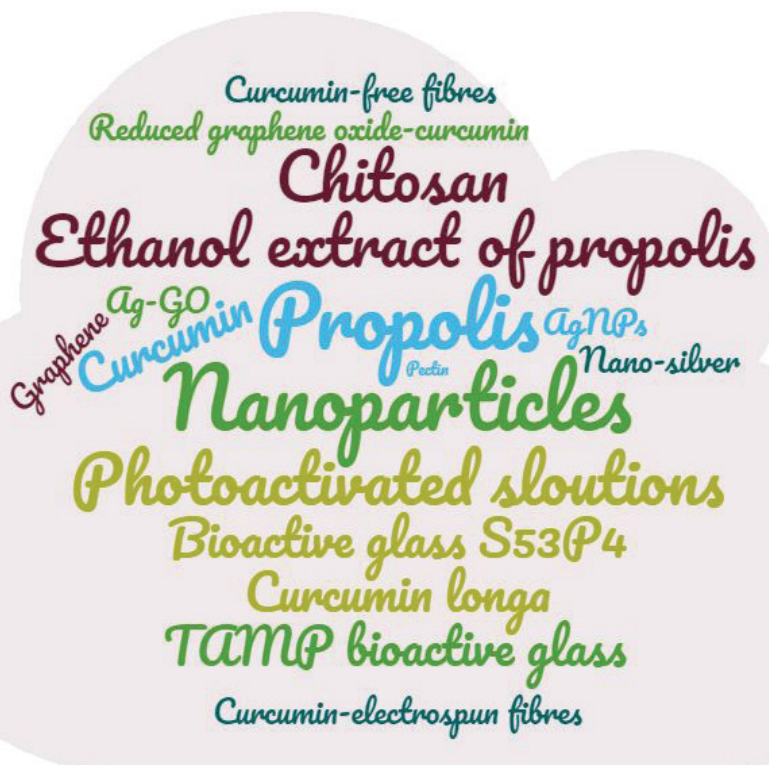

Fig. 2. Word cloud representing alternative antimicrobial materials and strategies for potential use in RET. The more a substance was researched, within this specific field, the larger it appears in the word cloud. Created by Wordclouds.com. 


\section{Natural materials and strategies}

\section{Propolis}

Propolis, also known as "bee glue", is a natural resinous substance crucial for both internal and external beehive protection (Ghisalberti, 1979; Grange and Davey, 1990). This resinous material is initially collected by bees (Apis mellifera) from exudates and plant buds, subsequently mixed with saliva secretions (bee enzymes) and wax (Ghisalberti, 1979; Grange and Davey, 1990). Historically, propolis has been used by on Egyptian, Greek and Roman traditional medicine since 300 BC (Khalil, 2006; Sforcin and Bankova, 2011).

Propolis comprises mainly resins, balsams, and wax in addition to amino acids, aromatic compounds, phenols, pollens, minerals, and vitamins (Ghisalberti, 1979; Grange and Davey, 1990; Uzel et al., 2005). The resinous portion is mainly composed of flavonoid pigments (well-known plant compounds) and is regarded the main active component, linked to propolis broad-spectrum antimicrobial activity (Ghisalberti, 1979; Grange and Davey, 1990). Additional therapeutic features include anti-oxidant, anti-cariogenic, and anti-inflammatory properties (Khalil, 2006; Marcucci, 1995; Uzel et al., 2005).

This resinous material has been highlighted as a promising natural additive to the chemical composition of toothpastes (Morawiec et al., 2013) and mouthwashes (Dodwad and Kukreja, 2011; Halboub et al., 2020). Possible avenues of propolis use in dentistry include prevention of dental caries and plaque formation (Koo et al., 2000), a cell preservation medium for avulsed teeth (Martin and Pileggi, 2004; Ozan et al., 2007), management of pulp exposures (Ahangari et al., 2012), and as an antimicrobial agent for the root canal system (El-Tayeb et al., 2019; Pagliarin et al., 2016).

Although propolis possesses various therapeutic properties, its chemical composition varies according to the country of origin, botanical source, and time of collection (Marcucci, 1995; Uzel et al., 2005). Clinically, the variation in chemical composition could ultimately result in a range of propolis therapeutic deficiencies and raises concerns in terms of quality control and batch-to-batch variability for standardised new drug development (Marcucci, 1995; Sforcin and Bankova, 2011). Despite such concerns, a standardised propolis extract, known as $\mathrm{EPP}-\mathrm{AF}^{\circledR}$, has been developed in Brazil (Berretta et al., 2012). Due to propolis impurities, a series of various purification and extraction methods are required, such as maceration or Soxhlet extraction (Ghisalberti, 1979). The use of strong solvents, such as ethanol or DMSO, during propolis extraction are reported and will have a negative effect the on cell viability and their regeneration potential, even at concentrations as low as $0.1 \%$ (Cunha et al., 2004; Sut et al., 2016). The lack of methodologically robust studies with detailed propolis chemical composition or its extraction method, lead to its limited clinical translation (Sforcin and Bankova, 2011). The use of nontoxic extraction solvents within well-controlled and designed comparative studies are required for further assessment of propolis as a promising material with potential clinical application in RET (Sut et al., 2016).

Propolis biocompatibility and regenerative potential towards soft and mineralised tissues have been reported (Ahangari et al., 2012; Al-Shaher et al., 2004). Propolis, at concentrations of $4 \mathrm{mg} / \mathrm{mL}$ or lower, was found to be at least 10 times less cytotoxic to fibroblasts of the dental pulp and periodontal ligament when compared with $\mathrm{Ca}(\mathrm{OH})_{2}$ (Al-Shaher et al., 2004). The use of propolis as a vehicle for $\mathrm{Ca}(\mathrm{OH})_{2}$ has also been suggested, with in vitro studies concluding efficient diffusion throughout the dentinal tubules, and possibly extending to the external root surface (Baranwal et al., 2017; Montero and Mori, 2012; de Rezende et al., 2008). Furthermore, animal studies utilising propolis paste as an intracanal medicament have shown promising results equal to or superior to TAP in-terms of soft and hard tissue deposition (El-Tayeb et al., 2019; Pagliarin et al., 2016). In vitro and in vivo studies utilising propolis as an intra-canal disinfectant agent are summarised in Table 1 and 2, respectively.

\section{Chitosan-based particles}

Chitosan (poly[1,4],-b-D-glucopyranosamine) is a cationic natural nontoxic biopolymer obtained by the alkaline deacetylation of chitin (Peter, 1995; Rabea et al., 2003). Chitin is the second most abundant natural polymer found in the exoskeleton of marine crustaceans such as shrimps and crab shells (Peter, 1995; Rabea et al., 2003).

Chitosan has a high nitrogen content with a strong chelating ability and great commercial interest (Rabea et al., 2003). On a production scale, chitosan can be produced in several forms, such as a paste or powder, with particles at the macro- or nano-scale (Agnihotri et al., 2004). Commercially, chitosan is available with an average molecular weight of 3,800 - 20,000 Daltons and 66 - $95 \%$ deacetylation (Agnihotri et al., 2004). Its versatile commercial applications include environmental, agricultural, food additive, and a hydrating agent in cosmetics (Peter, 1995; Rabea et al., 2003). Chitosan-based particles are regarded as an effective drug delivery system (Li et al., 2018), and tested as a vehicle for $\mathrm{Ca}(\mathrm{OH})_{2}$ or TAP with promising results such as improved stability and promoting a sustained release of medicament (Ballal et al., 2010; del Carpio-Perochena et al., 2017; Shaik et al., 2014). More recently, chitosan has been explored as an antimicrobial agent to disinfect the root canal system with proposed regenerative potential (Ducret et al., 2019; Palma et al., 2017).

This attractive research interest is linked to its unique biological properties such as biocompatibility, excellent bioadhesive, and broad-spectrum antimicrobial properties (Raafat and Sahl, 2009; Rabea et al., 2003; Shrestha et al., 2010). A postulated 
mechanism of chitosan antimicrobial activity is linked to the interaction between positively charged chitosan $\left(\mathrm{NH}_{3}^{+}\right.$groups of glucosamine) and negatively charged cell membrane causing a sequential of events, which alter the cell permeability and consequently cell death (Raafat and Sahl, 2009; Rabea et al., 2003). Additional advantages for extended clinical applications include abundance in nature, ease of modification, nontoxic, and low production cost (Peter, 1995; Rabea et al., 2003).

However, in spite of chitosan's proven biocompatibility and noncytotoxicity (Renard et al., 2020), its regenerative potential in dentistry remains controversial. Preliminary in vitro work demonstrated stimulation of dental pulp tissue formation, in terms of mesenchymal stem cells viability and deposition of dental pulp-like collagen matrix following the use of a novel chitosan-enriched fibrin hydrogel (Ducret et al., 2019). In-contrast, the clinical application of chitosan scaffolds in immature dog teeth with apical periodontitis resulted in no histologic evidence of pulp-dentine tissue regeneration nor newly formed mineralised tissue (Palma et al., 2017). The degradation process of chitosan under inflammatory conditions requires careful assessment prior to clinical translation (Palma et al., 2017; Yamada et al., 2014). In vitro studies utilising chitosan-based particles as an intra-canal disinfectant agent are summarised in Table 1.

Table 1a. In vitro and ex vivo studies of alternative antimicrobial strategies highlighting antimicrobial type, origin, study design, usage, study and control groups, assessment method and duration, microorganisms tested and main findings.

\begin{tabular}{|c|c|c|c|c|c|c|c|}
\hline $\begin{array}{c}\text { Alternative } \\
\text { antimicrobial/ } \\
\text { origin }\end{array}$ & $\begin{array}{l}\text { Study } \\
\text { design/ } \\
\text { usage }\end{array}$ & $\begin{array}{l}\text { Study } \\
\text { groups }\end{array}$ & $\begin{array}{l}\text { Control } \\
\text { groups }\end{array}$ & Microorganism & $\begin{array}{l}\text { Assessment } \\
\text { method/ } \\
\text { duration }\end{array}$ & Main findings & Reference \\
\hline $\begin{array}{c}\text { Propolis/ } \\
\text { Nature Home, } \\
\text { Amman, } \\
\text { Jordan }\end{array}$ & $\begin{array}{c}\begin{array}{c}\text { Human } \\
\text { dentine } \\
\text { block model }\end{array} \\
\text { Medicament }\end{array}$ & $\begin{array}{c}30 \% \\
\text { propolis } \\
\mathrm{Ca}(\mathrm{OH})_{2}\end{array}$ & $\begin{array}{c}\text { Sterile } \\
\text { uninoculated } \\
\text { broth }\end{array}$ & E. faecalis & $\begin{array}{c}\text { Microbiological } \\
\text { samples were } \\
\text { collected (paper } \\
\text { points, headstrom } \\
\text { files and disc } \\
\text { immersion), } \\
\text { incubated on agar } \\
\text { plates and CFUs } \\
\text { analysed } \\
1 \text { and } 2 \mathrm{~d} \\
\end{array}$ & $\begin{array}{l}\text { Propolis was } \\
\text { significantly } \\
\text { more effective } \\
\text { than } \mathrm{Ca}(\mathrm{OH})_{2} \\
\text { for short-term } \\
\text { application }\end{array}$ & $\begin{array}{l}\text { Awawdeh } \\
\text { et al. } \\
2009\end{array}$ \\
\hline Propolis & $\begin{array}{c}\begin{array}{c}\text { Human } \\
\text { dentine } \\
\text { block model }\end{array} \\
\text { Irrigant }\end{array}$ & $\begin{array}{c}\text { Propolis } \\
\text { MCJ } \\
2 \% \text { POVI } \\
2 \% \text { CHX gel } \\
\mathrm{Ca}(\mathrm{OH})_{2}\end{array}$ & Saline & E. faecalis & $\begin{array}{l}\text { Dentine shavings } \\
\text { were collected } \\
\text { (200 and } 400 \mu \mathrm{m} \\
\text { depths), cultured } \\
\text { on TS agar } \\
\text { plates and CFUs } \\
\text { analysed } \\
21 \mathrm{~d}\end{array}$ & \begin{tabular}{|c|} 
CHX \\
produced \\
the highest \\
antimicrobial \\
efficacy \\
followed \\
by POVI, \\
propolis and \\
MCJ. Ca $(\mathrm{OH})_{2}$ \\
was least \\
effective \\
\end{tabular} & $\begin{array}{c}\text { Kandaswamy } \\
\text { et al., } \\
2010\end{array}$ \\
\hline $\begin{array}{c}\text { Propolis/ } \\
\text { Apis flora, } \\
\text { Ribeirão Preto, } \\
\text { Brazil }\end{array}$ & $\begin{array}{c}\text { Human root } \\
\text { model } \\
\text { Irrigant }\end{array}$ & $\begin{array}{l}12 \% \\
\text { propolis } \\
\text { glycolic } \\
\text { extract }\end{array}$ & Saline & E. coli & $\begin{array}{l}\text { Microbiological } \\
\text { samples were } \\
\text { collected, } \\
\text { incubated on agar } \\
\text { plates and CFUs } \\
\text { analysed }\end{array}$ & $\begin{array}{l}\text { Propolis was } \\
\text { effective to } \\
\text { completely } \\
\text { eliminate } \\
\text { E. coli and } \\
\text { reduce the } \\
\text { amount of } \\
\text { endotoxins } \\
\end{array}$ & $\begin{array}{l}\text { Valera } \\
\text { et al., } \\
2010\end{array}$ \\
\hline $\begin{array}{l}\text { Propolis/ } \\
\text { Turkey } \\
\text { (northeast } \\
\text { and northwest } \\
\text { areas) }\end{array}$ & $\begin{array}{c}\begin{array}{c}\text { Human } \\
\text { dentine } \\
\text { block model }\end{array} \\
\text { Irrigant }\end{array}$ & $\begin{array}{c}\text { EEP } \\
2 \% \text { CHX } \\
\text { solution } \\
\mathrm{Ca}(\mathrm{OH})_{2}\end{array}$ & $\begin{array}{c}96 \% \text { ethanol } \\
\text { PBS }\end{array}$ & E. faecalis & $\begin{array}{c}\text { Dentine shavings } \\
\text { were collected } \\
(300 \mu \mathrm{m} \text { depth), } \\
\text { cultured on TS } \\
\text { agar plates and } \\
\text { CFUs analysed } \\
7 \mathrm{~d} \\
\end{array}$ & $\begin{array}{c}\text { Propolis } \\
\text { antimicrobial } \\
\text { efficacy was } \\
\text { higher than } \\
\mathrm{Ca}(\mathrm{OH})_{2} \text { and } \\
\text { lower than } \\
\mathrm{CHX}\end{array}$ & $\begin{array}{l}\text { Kayaoglu } \\
\text { et al., } \\
2011\end{array}$ \\
\hline $\begin{array}{c}\text { Propolis/ } \\
\text { Calgary gold } \\
\text { bee products, } \\
\text { Canada }\end{array}$ & $\begin{array}{l}\begin{array}{c}\text { Human root } \\
\text { model }\end{array} \\
\text { Medicament }\end{array}$ & $\begin{array}{l}\mathrm{Ca}(\mathrm{OH})_{2} \\
\text { TAP } \\
\text { EEP } \\
\text { ethanol }\end{array}$ & Saline & E. faecalis & $\begin{array}{c}\text { Percentage } \\
\text { reduction } \\
\text { in colony counts } \\
1,2 \text { and } 7 \mathrm{~d}\end{array}$ & $\begin{array}{c}\text { Propolis } \\
\text { was more } \\
\text { effective than } \\
\text { TAP at day } 2 \\
\text { and equally } \\
\text { effective at } \\
\text { day } 7\end{array}$ & $\begin{array}{l}\text { Madhubala } \\
\text { et al., } \\
2011\end{array}$ \\
\hline
\end{tabular}




\section{Curcumin}

Curcumin (diferuloylmethane), a dimeric derivative of ferulic acid, is the main bioactive substance of C. longa (turmeric), a wellknown oriental spice (Adamczak et al., 2020). Curcumin pigment, a distinctive yellow-orange colouring matter of plant origin, was isolated back in 1842 by Vogel and Pelletier from the rhizomes of C. longa, originating from the ginger family tree native to South Asia (Adamczak et al., 2020; Hewlings and Kalman, 2017).

Curcumin possesses a wide spectrum of bioactive and therapeutic properties such as antibacterial, antifungal, and antiviral properties (Praditya et al., 2019; Rai et al., 2008). Antioxidant and antiinflammatory activities are also documented in the literature (Hewlings and Kalman, 2017; Rai et al., 2020; Sinjari et al., 2019). The antimicrobial action of curcumins is attributed to their ability to damage the bacterial cell membrane through the suppression of bacterial cytokinesis, the induction of filamentation, and the inhibition of the FtsZ assembly dynamics in the Z-ring (Rai et al., 2008). Inhibition of cellular proliferation and alterations of gene expression are also reported to be behind the bactericidal mechanisms of curcumins (Rai et al., 2008; Rai et al., 2020; Tyagi et al., 2015).

In the past, the clinical usage of curcumins was limited due to their poor water solubility, low oral bioavailability, and rapid metabolism (Chang et al., 2018; Sinjari et al., 2019). The use of liposomes, in order to solubilise curcumin phospholipidic bilayer, has been suggested to enhance curcumin delivery and improve its therapeutic efficiency (Chang et al., 2018; Sinjari et al., 2019). Sinjari et al. (2019) closely assessed the direct contact of human DPSCs with nanocarrier curcumin-loaded

Table 1b. In vitro and ex vivo studies of alternative antimicrobial strategies highlighting antimicrobial type, origin, study design, usage, study and control groups, assessment method and duration, microorganisms tested and main findings.

\begin{tabular}{|c|c|c|c|c|c|c|c|}
\hline $\begin{array}{c}\text { Alternative } \\
\text { antimicrobial/ } \\
\text { origin }\end{array}$ & $\begin{array}{l}\text { Study } \\
\text { design/ } \\
\text { usage }\end{array}$ & $\begin{array}{l}\text { Study } \\
\text { groups }\end{array}$ & $\begin{array}{l}\text { Control } \\
\text { groups }\end{array}$ & Microorganism & $\begin{array}{c}\text { Assessment } \\
\text { method/ } \\
\text { duration }\end{array}$ & Main findings & Reference \\
\hline $\begin{array}{l}\text { Propolis/ } \\
\text { Beehives of } \\
\text { Najaf Abad, } \\
\text { Esfahan }\end{array}$ & $\begin{array}{l}\text { Human root } \\
\text { model }\end{array}$ & $\begin{array}{c}\mathrm{EEP} \\
\mathrm{Ca}(\mathrm{OH})_{2} \\
\text { ethanol }\end{array}$ & $\begin{array}{c}\text { No } \\
\text { medicament } \\
\text { Sterile } \\
\text { samples }\end{array}$ & E. faecalis & $\begin{array}{l}\text { Microbiological } \\
\text { samples were } \\
\text { collected with a } \\
\text { piezoreamer, plated } \\
\text { and CFU analysed. } \\
\text { MIC was also } \\
\text { measured using } \\
\text { dilution methods } \\
7 \mathrm{~d}\end{array}$ & $\begin{array}{l}\text { CFUs and MIC } \\
\text { of propolis were } \\
\text { significantly less } \\
\text { than } \mathrm{Ca}(\mathrm{OH})_{2}\end{array}$ & $\begin{array}{c}\text { Zare } \\
\text { Jahromi } \\
\text { et al., } \\
2012\end{array}$ \\
\hline $\begin{array}{l}\text { Propolis/ } \\
\text { RK's Aroma } \\
\text { Products, } \\
\text { Mumbai }\end{array}$ & $\begin{array}{l}\text { Human root } \\
\text { model }\end{array}$ & $\begin{array}{c}\text { Propolis } \\
2 \% \mathrm{CHX} \\
\text { gel } \\
\mathrm{Ca}(\mathrm{OH})_{2}\end{array}$ & Saline & E. faecalis & $\begin{array}{l}\text { Dentine shavings } \\
\text { were collected } \\
\text { (depth of } 400 \mu \mathrm{m} \text { ), } \\
\text { cultured on TS agar } \\
\text { and CFUs analysed } \\
1,3 \text { and } 5 \mathrm{~d}\end{array}$ & $\begin{array}{c}\text { Propolis } \\
\text { had greater } \\
\text { antimicrobial } \\
\text { efficacy than } \\
\mathrm{Ca}(\mathrm{OH})_{2} \text { on day } 1 \text {, } \\
\text { with no significant } \\
\text { difference in } \\
\text { subsequent days }\end{array}$ & $\begin{array}{c}\text { Bhandari } \\
\text { et al., } \\
2014\end{array}$ \\
\hline $\begin{array}{c}\text { Propolis/ } \\
\text { Natural Bee } \\
\text { Health } \\
\text { Industry, Lima, } \\
\text { Peru }\end{array}$ & $\begin{array}{c}\text { Human root } \\
\text { model } \\
\text { Irrigant }\end{array}$ & $\begin{array}{c}\mathrm{Ca}(\mathrm{OH})_{2} \\
\text { Propolis } \\
2 \% \mathrm{CHX} \\
\text { gel }\end{array}$ & Saline & $\begin{array}{l}\text { E. faecalis } \\
\quad \text { and } \\
\text { C. albicans }\end{array}$ & $\begin{array}{c}\text { Dentine shavings } \\
\text { were collected } \\
\text { (100 and } 200 \mu \mathrm{m} \\
\text { depths), cultured } \\
\text { on agar blood or } \\
\text { agar Sabouraud } \\
\text { plates and CFUs } \\
\text { analysed } \\
14 \mathrm{~d}\end{array}$ & $\begin{array}{l}\text { Propolis and CHX } \\
\text { were the most } \\
\text { effective against } E \text {. } \\
\text { faecalis. However, } \\
\mathrm{CHX} \text { had the } \\
\text { highest antifungal } \\
\text { activity }\end{array}$ & $\begin{array}{c}\text { Carbajal } \\
\text { Mejía, } \\
2014\end{array}$ \\
\hline $\begin{array}{c}\text { Propolis/ } \\
\text { Stakich, Royal } \\
\text { Oak, Michigan, } \\
\text { USA }\end{array}$ & $\begin{array}{l}\text { Human root } \\
\text { model }\end{array}$ & $\begin{array}{c}95 \% \\
\text { propolis } \\
\text { TAP } \\
2 \% \text { CHX } \\
\text { gel } \\
\mathrm{Ca}(\mathrm{OH})_{2}\end{array}$ & Saline & C. albicans & $\begin{array}{l}\text { Dentine shavings } \\
\text { were collected (200 } \\
\text { and } 400 \mu \mathrm{m} \text { depths) } \\
\text { and CFUs analysed } \\
1 \text { and } 7 \mathrm{~d}\end{array}$ & $\begin{array}{l}\text { Propolis was less } \\
\text { effective on day } \\
1 \text { and equally } \\
\text { effective to other } \\
\text { medicaments on } \\
\text { day } 7\end{array}$ & $\begin{array}{l}\text { Chua } \\
\text { et al., } \\
2014\end{array}$ \\
\hline $\begin{array}{c}\text { Propolis/ } \\
\text { Herbal } \\
\text { Biosolutions, } \\
\text { Delhi } \\
\text { C. longa/ } \\
\text { RYM exports, } \\
\text { Mumbai, India }\end{array}$ & $\begin{array}{c}\begin{array}{c}\text { Human } \\
\text { dentine } \\
\text { block model }\end{array} \\
\text { Medicament }\end{array}$ & $\begin{array}{l}2 \% \mathrm{CHX} \\
\text { gel honey } \\
\text { Aloe vera } \\
\text { gel } \\
20 \% \mathrm{C} \text {. } \\
\text { longa gel } \\
11 \% \mathrm{EEP} \\
\mathrm{Ca}(\mathrm{OH})_{2}\end{array}$ & Saline & E. faecalis & $\begin{array}{c}\text { Dentine shavings } \\
\text { were collected (200 } \\
\text { and } 400 \mu \mathrm{m} \text { depths) } \\
\text { and CFUs were } \\
\text { analysed. } \\
1,3 \text { and } 5 \mathrm{~d}\end{array}$ & $\begin{array}{l}2 \% \text { CHX gel was } \\
\text { most effective } \\
\text { followed by } \\
\text { propolis and } C . \\
\text { longa }\end{array}$ & $\begin{array}{c}\text { Vasudeva } \\
\text { et al., } \\
2017\end{array}$ \\
\hline
\end{tabular}


liposome in the presence of hydrophilic monomers (2-hydroxyethyl methacrylate). Quantitative cytokine release assessment showed a decreased secretion of tested pro-inflammatory cytokines (interleukin 6 , interleukin 8, interferon-gamma, and monocyte chemoattractant protein-1), in response to curcumin liposome nanocarriers. The authors concluded that curcumin liposome nanocarriers had stimulated DPSCs proliferation and reduced inflammation via the NFkB/ERK/pERK pathways, but did not induce odontoblastic differentiation (Sinjari et al., 2019). Various loading and encapsulation mechanisms for curcumin delivery such as nanoemulsion, nanosuspension, lipid nanoparticles, and hydrogel nanoparticles were further investigated in the literature (Dutta and Ikiki, 2013; Rai et al., 2020).

Despite the above-mentioned therapeutic and bioactive properties, limited studies were conducted within the dental field, particularly in RET (Neelakantan et al., 2013; Yadav et al., 2018). Emerging in vitro studies, with promising results, expanding the use of curcumins as an intra-canal disinfectant agent are summarised in Table 1.

\section{AgNPs}

Inorganic metals, in their standard or ionic forms such as $\mathrm{Ag}$ or $\mathrm{Ag}^{+}$, are regarded as antibiotic alternatives due to their broad-spectrum bactericidal effects coupled with the unlikely possibility of developing antibiotic-resistant bacterial strains (Oei et al., 2012;
Rai et al., 2009). Specifically, AgNPs have gained recent popularity owing to their distinctive physical and biochemical properties (Bapat et al., 2018; Rai et al., 2009), synergistic antibiotic effect (Fayaz et al., 2010), biocompatibility (Franková et al., 2016; GomesFilho et al., 2010), and antimicrobial properties (Lara et al., 2010; Rai et al., 2009). The ability of AgNPs to disrupt dental biofilms and prevent bacterial adhesion are also advantageous (Wu et al., 2014).

The incorporation of silver particles within dental materials is not new and has been practiced for decades since the use of silver-containing dental amalgam (Noronha et al., 2017). However, with advanced nanotechnology, AgNPs have gained considerable attention (Bapat et al., 2018; Noronha et al., 2017). The incorporation of AgNPs within a diverse range of dental materials have been proposed, such as composite resins (Cheng et al., 2012; Durner et al., 2011), dental implant coating (Cao et al., 2011; Wang et al., 2013), calcium silicates cements (Fan et al., 2014; Samiei et al., 2013), endodontic sealers (Vilela Teixeira et al., 2017), and intra-canal disinfectant agents (Afkhami et al., 2017; Afkhami et al., 2015; Moazami et al., 2018; Wu et al., 2014).

The antimicrobial mode of action of AgNPs is largely associated with the release of $\mathrm{Ag}^{+}$ions, which consequently penetrate the cell membrane and interact with various cellular components, resulting in inhibition of cell replication and eventually cell death (Bapat et al., 2018; Rai et al., 2009). An immediate

Table 1c. In vitro and ex vivo studies of alternative antimicrobial strategies highlighting antimicrobial type, origin, study design, usage, study and control groups, assessment method and duration, microorganisms tested and main findings.

\begin{tabular}{|c|c|c|c|c|c|c|c|}
\hline $\begin{array}{c}\text { Alternative } \\
\text { antimicrobial/ } \\
\text { origin }\end{array}$ & $\begin{array}{l}\text { Study } \\
\text { design/ } \\
\text { usage }\end{array}$ & $\begin{array}{l}\text { Study } \\
\text { groups }\end{array}$ & $\begin{array}{l}\text { Control } \\
\text { groups }\end{array}$ & Microorganism & $\begin{array}{c}\text { Assessment } \\
\text { method/ } \\
\text { duration }\end{array}$ & Main findings & Reference \\
\hline $\begin{array}{c}\text { Chitosan/ } \\
\text { Sigma-Aldrich } \\
\text { Inc. }\end{array}$ & $\begin{array}{c}\text { Well } \\
\text { plates } \\
\text { irrigant }\end{array}$ & $\begin{array}{l}\text { Chitosan- } \\
\text { nanoparticle } \\
\text { Zinc oxide- } \\
\text { nanoparticle }\end{array}$ & - & $\begin{array}{l}2 \text { strains of } \\
\text { E. faecalis in } \\
\text { planktonic and } \\
\text { biofilm forms }\end{array}$ & $\begin{array}{l}\text { LIVE/DEAD } \\
\text { staining/confocal } \\
\text { microscopy/ } \\
\text { CFUs } \\
90 \mathrm{~d} \\
\end{array}$ & $\begin{array}{c}\text { The incorporation of } \\
\text { nanoparticles enhanced } \\
\text { antimicrobial efficacy } \\
\text { with retained aging } \\
\text { potential }\end{array}$ & $\begin{array}{l}\text { Shrestha } \\
\text { et al., } \\
2010\end{array}$ \\
\hline $\begin{array}{c}\text { Chitosan/ } \\
\text { Acros Organics }\end{array}$ & $\begin{array}{l}\text { Human } \\
\text { root } \\
\text { model } \\
\text { irrigant }\end{array}$ & $\begin{array}{c}15 \% \text { EDTA } \\
0.2 \% \\
\text { chitosan } \\
10 \% \text { citric } \\
\text { acid } \\
1 \% \text { acetic } \\
\text { acid } \\
\end{array}$ & $\begin{array}{c}\text { No final } \\
\text { irrigation }\end{array}$ & - & $\begin{array}{l}\text { SEM/ } \\
\text { atomic absorption } \\
\text { spectrophotometry }\end{array}$ & $\begin{array}{l}0.2 \% \text { chitosan was } \\
\text { similar to } 15 \% \text { EDTA } \\
\text { in terms of smear } \\
\text { layer and dentine } \\
\text { demineralisation }\end{array}$ & $\begin{array}{l}\text { Silva } \\
\text { et al., } \\
2013\end{array}$ \\
\hline $\begin{array}{l}\text { Chitosan/ } \\
\text { Mahtani } \\
\text { Chitosan Pvt. } \\
\text { Ltd Veraval, } \\
\text { India }\end{array}$ & $\begin{array}{c}\text { Human } \\
\text { root } \\
\text { model } \\
\text { irrigant }\end{array}$ & $\begin{array}{c}0.25 \% \\
\text { chitosan } \\
0.5 \% \\
\text { chitosan } \\
2 \% \mathrm{CHX} \\
3 \% \mathrm{NaOCl}\end{array}$ & Saline & $\begin{array}{l}\text { E. faecalis } \\
\text { and } \\
\text { C. albicans } \\
\text { biofilms }\end{array}$ & $\begin{array}{c}\text { Agar-well } \\
\text { diffusion method/ } \\
\text { MIC/ biofilm } \\
\text { susceptibility } \\
\text { assay/ } \\
\text { SEM/ } \\
\text { cytotoxicity assay/ } \\
\text { CFUs }\end{array}$ & $\begin{array}{l}\text { Similar antimicrobial } \\
\text { efficacy was seen } \\
\text { between study groups. } \\
\text { However, chitosan } \\
\text { showed significant less } \\
\text { toxicity then } \mathrm{NaOCl}\end{array}$ & $\begin{array}{l}\text { Yadav } \\
\text { et al., } \\
2017\end{array}$ \\
\hline \begin{tabular}{c|} 
Curcumin/ \\
Biopurify \\
Phytochemicals \\
Ltd., Sichuan, \\
China
\end{tabular} & $\begin{array}{l}\text { Human } \\
\text { root } \\
\text { model }\end{array}$ & $\begin{array}{c}\text { Curcumin } \\
2 \% \mathrm{CHX} \\
3 \% \mathrm{NaOCl}\end{array}$ & PBS & $\begin{array}{l}\text { E. faecalis } \\
\text { biofilms }\end{array}$ & $\begin{array}{l}\mathrm{MIC} / \mathrm{MBC} / \\
\text { CFUs } \\
2^{\text {nd }} \mathrm{d}, 2^{\text {nd }} \text { and } \\
8^{\text {th }} \text { weeks }\end{array}$ & $\begin{array}{c}\text { Curcumin } \\
\text { antimicrobial efficacy } \\
\text { was similar to } 3 \% \\
\mathrm{NaOCl} \text { at } 2 \mathrm{~d} \text { and } 2^{\text {nd }} \\
\text { week, and inferior to } \\
\mathrm{CHX} \\
\text { at } 8^{\text {th }} \text { week }\end{array}$ & $\begin{array}{l}\text { Neelakantan } \\
\text { et al., } \\
2013\end{array}$ \\
\hline
\end{tabular}


bactericidal antimicrobial effect of AgNPs has been shown against several drug-resistant and drugsusceptible bacteria possibly through inhibiting cell wall, protein, and nucleic acid synthesis (Lara et al., 2010).

However, there are some cytotoxicity concerns to the use of AgNPs, mainly related to adverse events of free $\mathrm{Ag}^{+}$. Such concerns are controversial and at an early stage of research for sound conclusions to be drawn. Franková et al. (2016) assessed AgNPs against human dermal fibroblasts and epidermal keratinocytes. They concluded that AgNPs inhibit the production of pro-inflammatory cytokines and contributed positively towards the wound-healing process. Quantitative elution testing and qualitative on-growth of human osteoblasts also revealed no cytotoxicity following the use of $1 \%$ nano-silver loaded bone cement in vitro (Alt et al., 2004). Indeed, it was proven that AgNPs biocompatibility is concentration dependent with increased cytotoxicity at higher concentrations (Gomes-Filho et al., 2010; Newby et al., 2011). In addition to material concentration, material-specific characteristics (Cao et al., 2011) and incorporation methods (Fan et al., 2014) also determine the cytotoxicity of AgNPs. To investigate the latter, the cytotoxicity of nano-silver incorporated using two methods (template and absorption method) to mesoporous calcium silicate against human bone marrow mesenchymal cells was assessed (Fan et al., 2014). Results indicated no obvious cytotoxicity following the adoption of the template method, in contrast to significant cytotoxicity associated with the absorption method (Fan et al., 2014).

Despite the significant advantages of silverbased materials, potential adverse effects have been reported. Argyria, an irreversible skin pigmentation, is a well-known silver-related side effect (Drake and Hazelwood, 2005). Furthermore, potential tooth discoloration is also of concern and requires careful investigation prior to clinical usage (Afkhami et al., 2015). In vitro spectrophotometric analysis showed no significant colour changes between AgNPs added to $\mathrm{Ca}(\mathrm{OH})_{2}$ compared to $\mathrm{Ca}(\mathrm{OH})_{2}$ alone when used as an intra-canal medicament (Afkhami et al., 2017). In contrast, spectrophotometric analysis of AgNPs

Table 1d. In vitro and ex vivo studies of alternative antimicrobial strategies highlighting antimicrobial type, origin, study design, usage, study and control groups, assessment method and duration, microorganisms tested and main findings.

\begin{tabular}{|c|c|c|c|c|c|c|c|}
\hline $\begin{array}{c}\text { Alternative } \\
\text { antimicrobial/ } \\
\text { origin }\end{array}$ & $\begin{array}{l}\text { Study } \\
\text { design/ } \\
\text { usage }\end{array}$ & Study groups & $\begin{array}{l}\text { Control } \\
\text { groups }\end{array}$ & Microorganism & $\begin{array}{l}\text { Assessment } \\
\text { method/ } \\
\text { duration }\end{array}$ & Main findings & Reference \\
\hline $\begin{array}{l}\text { Photoactivated } \\
\text { curcumin/ } \\
20 \mu \mathrm{mol} / \mathrm{L} / \\
\text { Sigma-Aldrich, } \\
\text { St Louis, MO, } \\
\text { USA }\end{array}$ & $\begin{array}{l}\text { Human } \\
\text { root } \\
\text { model }\end{array}$ & Curcumin & $\begin{array}{l}\text { Physiological } \\
\text { solution }\end{array}$ & $\begin{array}{l}\text { E. faecalis } \\
\text { biofilms }\end{array}$ & 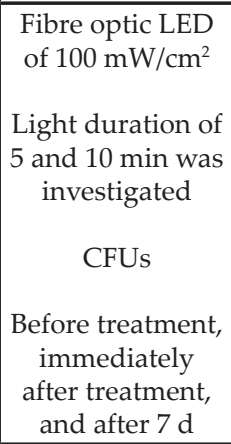 & $\begin{array}{l}\text { Photoactivated } \\
\text { curcumin } \\
\text { reduced, however } \\
\text { did not eliminate, } \\
\text { bacterial count }\end{array}$ & $\begin{array}{c}\text { da Frota } \\
\text { et al., } \\
2015\end{array}$ \\
\hline $\begin{array}{l}\text { Curcumin/ } \\
2.5 \mathrm{mg} / \mathrm{mL}\end{array}$ & $\begin{array}{l}\text { Human } \\
\text { root } \\
\text { model }\end{array}$ & $\begin{array}{l}\text { Curcumin } \\
3 \% \mathrm{NaOCI}\end{array}$ & Saline & $\begin{array}{l}\text { E. faecalis } \\
\text { biofilms }\end{array}$ & $\begin{array}{c}\text { Ultrasonic } \\
\text { activated for } 30 \mathrm{~s} \\
\text { cycles for } 4 \mathrm{~min} / \\
\text { photoactivated } \\
\text { with blue light of } \\
1200 \mathrm{~mW} / \mathrm{cm}^{2} \text { for } \\
4 \mathrm{~min} / \\
\mathrm{SEM} / \\
\text { CFUs }\end{array}$ & $\begin{array}{l}\text { Photoactivated } \\
\text { curcumin } \\
\text { produced the } \\
\text { maximum } \\
\text { elimination of } \\
\text { biofilm bacteria }\end{array}$ & $\begin{array}{l}\text { Neelakantan } \\
\text { et al., } \\
2015\end{array}$ \\
\hline $\begin{array}{l}\text { Photoactivated } \\
\text { curcumin/ } \\
2.5 \mathrm{mg} / \mathrm{mL}\end{array}$ & $\begin{array}{l}\text { Human } \\
\text { root } \\
\text { model }\end{array}$ & $\begin{array}{c}\text { Photoactivated } \\
\text { curcumin } \\
\text { TAP } \\
\text { DAP } \\
2 \% \mathrm{CHX} \\
\mathrm{Ca}(\mathrm{OH})_{2}\end{array}$ & $\begin{array}{c}\text { No } \\
\text { medicament }\end{array}$ & $\begin{array}{l}\text { E. faecalis } \\
\text { biofilm }\end{array}$ & $\begin{array}{l}\text { LIVE/DEAD } \\
\text { staining/ } \\
\text { CLSM/ } \\
\text { CFUs } \\
14 \mathrm{~d}\end{array}$ & $\begin{array}{l}\text { Photoactivated } \\
\text { curcumin } \\
\text { produced superior } \\
\text { antimicrobial } \\
\text { and antibiofilm } \\
\text { activity }\end{array}$ & $\begin{array}{c}\text { Devaraj } \\
\text { et al., } \\
2016\end{array}$ \\
\hline $\begin{array}{c}\text { Curcumin/ } \\
2.5 \text { and } 5.0 \mathrm{mg} / \\
\mathrm{mL}\end{array}$ & $\begin{array}{c}\text { Well } \\
\text { plates } \\
\text { irrigant }\end{array}$ & $\begin{array}{c}\text { Curcumin- } \\
\text { electrospun } \\
\text { modified } \\
\text { fibres } \\
\text { Curcumin-free } \\
\text { fibres } \\
2 \% \mathrm{CHX} \\
1 \% \mathrm{NaOCl} \\
\text { TAP }\end{array}$ & Saline & $\begin{array}{l}\text { A. naeslundii } \\
\text { biofilms }\end{array}$ & CFUs & $\begin{array}{l}\text { Photoactivated } \\
\text { curcumin-based } \\
\text { medicaments } \\
\text { showed a high } \\
\text { antibiofilm } \\
\text { activity when } \\
\text { used at low } \\
\text { concentrations }\end{array}$ & $\begin{array}{l}\text { Sotomil } \\
\text { et al., } \\
2019\end{array}$ \\
\hline
\end{tabular}


coated with imidazolium as a root canal irrigant resulted in statistically significant darker colour changes in comparison with the control groups (5.25\% NaOCl, $2 \% \mathrm{CHX}$, and normal saline) (Moazami et al., 2018). The colour change caused by AgNPs could be linked mostly to the direct contact of the irrigant material with the coronal dentine, which is practically difficult to control during irrigation. Taking the above results into consideration, wellcontrolled clinical studies are warranted for sound conclusions and clinical recommendations. Emerging studies utilising AgNPs within the endodontic field as an intra-canal disinfectant agent are summarised in Table 1.

\section{Graphene-based materials}

Graphene is a recently developed material made of densely packed carbon atoms arranged as a twodimensional monolayer sheet forming a benzene hexagonal structure (Novoselov et al., 2004). Examples of graphene derivatives include pristine graphene, graphene oxide, reduced graphene oxide, few-layered graphene, ultrathin graphite, graphene nanosheets, and graphene-based nanocomposites (Guazzo et al., 2018; Tahriri et al., 2019). These different derivatives mainly differ in the material size, number of layers, and specific surface characteristics (Guazzo et al., 2018).

Graphene and its derivatives have a wide range of biomedical applications due to their unique mechanical, electrochemical, and physical properties such as mechanical strength, high surface area, thermal stability, and unique surface characteristics (Guazzo et al., 2018; Tahriri et al., 2019). Furthermore, their ability to combine and functionalise with various materials and bioactive molecules to enhance or alter specific properties for distinctive applications is of interest (Guazzo et al., 2018).

Graphene-based materials also have broadspectrum, yet variable, antimicrobial properties (Al-Jumaili et al., 2017; Guazzo et al., 2018). Its antimicrobial mode of action is explained through different mechanisms.

1. A sharp knife-edge cutting effect that consequently leads to cell membrane rupture, mechanical stress induction, and phospholipids extraction

Table 1e. In vitro and ex vivo studies of alternative antimicrobial strategies highlighting antimicrobial type, origin, study design, usage, study and control groups, assessment method and duration, microorganisms tested and main findings.

\begin{tabular}{|c|c|c|c|c|c|c|c|}
\hline $\begin{array}{c}\text { Alternative } \\
\text { antimicrobial/ } \\
\text { origin }\end{array}$ & $\begin{array}{l}\text { Study } \\
\text { design/ } \\
\text { usage }\end{array}$ & $\begin{array}{l}\text { Study } \\
\text { groups }\end{array}$ & $\begin{array}{l}\text { Control } \\
\text { groups }\end{array}$ & Microorganism & $\begin{array}{l}\text { Assessment } \\
\text { method/ } \\
\text { duration }\end{array}$ & Main findings & Reference \\
\hline $\begin{array}{c}\text { C. longa } \\
\text { extract/ } \\
\text { Curenext, } \\
\text { Abbott, India }\end{array}$ & $\begin{array}{l}\text { Human root } \\
\text { model } \\
\text { Medicament }\end{array}$ & $\begin{array}{c}\text { C. longa } \\
\text { extract } \\
1 \% \mathrm{CHX} \text { gel } \\
\mathrm{Ca}(\mathrm{OH})_{2}\end{array}$ & - & $\begin{array}{l}\text { E. faecalis } \\
\text { biofilms }\end{array}$ & $\begin{array}{l}\text { CFUs } \\
24 \mathrm{~h}\end{array}$ & $\begin{array}{c}\text { CHX gel showed } \\
\text { highest antimicrobial } \\
\text { efficacy followed } \\
\text { by C. longa extract, } \\
\text { while } \mathrm{Ca}(\mathrm{OH})_{2} \text { was } \\
\text { least effective }\end{array}$ & $\begin{array}{l}\text { Yadav } \\
\text { et al., } \\
2018\end{array}$ \\
\hline $\begin{array}{l}\text { Nano-silver } \\
\text { liquid form/ } \\
\text { Nanocid } \\
\text { Company, } \\
\text { Tehran, Iran }\end{array}$ & $\begin{array}{c}\text { Test } \\
\text { tubes and } \\
\text { inoculated } \\
\text { plates } \\
\text { Irrigant }\end{array}$ & $\begin{array}{c}\text { Nano-silver } \\
2 \% \mathrm{CHX} \\
5.25 \% \\
\mathrm{NaOCl}\end{array}$ & $\begin{array}{l}\text { Vancomycin } \\
\text { bacterial } \\
\text { susceptibility } \\
\text { papers } \\
\text { Saline } \\
\end{array}$ & E. faecalis & $\begin{array}{c}\text { MIC/ } \\
\text { agar diffusion/ } \\
\text { zones of inhibition } \\
6,18,24 \text { and } 48 \mathrm{~h} \\
\text { of incubation }\end{array}$ & $\begin{array}{c}\text { Nano-silver showed } \\
\text { equal bactericidal } \\
\text { action as } 5.25 \% \\
\text { NaOCI }\end{array}$ & $\begin{array}{l}\text { Lotfi } \\
\text { et al., } \\
2011\end{array}$ \\
\hline $\begin{array}{l}\text { Silver nitrate/ } \\
\text { Factory of } \\
\text { Shanghai } \\
\text { Chemical } \\
\text { Reagent Ltd, } \\
\text { China }\end{array}$ & $\begin{array}{l}\text { Longitudinal } \\
\text { split root } \\
\text { human teeth } \\
\text { model }\end{array}$ & $\begin{array}{l}\text { Nano-silver } \\
\text { incorporated } \\
\text { mesoporous } \\
\text { calcium } \\
\text { silicate }\end{array}$ & $\begin{array}{c}\mathrm{Ca}(\mathrm{OH})_{2} \\
\text { deionised } \\
\text { water }\end{array}$ & E. faecalis & $\begin{array}{c}\text { LIVE/DEAD } \\
\text { staining/ cell } \\
\text { counting kit-8 } \\
\text { Variable }\end{array}$ & $\begin{array}{l}\text { The incorporation } \\
\text { of Ag particles } \\
\text { resulted in enhanced } \\
\text { antibacterial } \\
\text { efficacy and } \\
\text { reduced bacterial } \\
\text { colonisation }\end{array}$ & $\begin{array}{l}\text { Fan } \\
\text { et al., } \\
2014\end{array}$ \\
\hline $\begin{array}{c}\text { AgNPs/ } \\
\text { Huzheng } \\
\text { Nano } \\
\text { Technology } \\
\text { Ltd, } \\
\text { Shanghai, } \\
\text { China }\end{array}$ & $\begin{array}{c}\text { Longitudinal } \\
\text { split root } \\
\text { human teeth } \\
\text { model } \\
\text { Irrigant/ } \\
\text { medicament }\end{array}$ & $\begin{array}{c}\text { Stage 1; } \\
0.1 \% \\
\text { AgNPs } \\
\text { solution } \\
2 \% \mathrm{NaOCl} \\
\text { Stage } 2 ; \\
0.01 \% \\
\text { AgNPs gel } \\
0.02 \% \\
\text { AgNPs gel } \\
\mathrm{Ca}(\mathrm{OH})_{2} \\
\end{array}$ & Sterile saline & E. faecalis & $\begin{array}{l}\text { SEM/ LIVE/DEAD } \\
\text { staining / CLSM } \\
\text { Stage 1; for } 2 \mathrm{~min} \\
\text { Stage 2; for } 7 \mathrm{~d}\end{array}$ & $\begin{array}{l}0.02 \% \text { AgNPs gel } \\
\text { as a medicament } \\
\text { resulted in } \\
\text { significant reduction } \\
\text { in residual biofilms }\end{array}$ & $\begin{array}{l}\text { Wu } \\
\text { et al., } \\
2014\end{array}$ \\
\hline Ag-GO & $\begin{array}{c}\text { Human root } \\
\text { model } \\
\text { Irrigant }\end{array}$ & $\begin{array}{c}1 \% \mathrm{NaOCl} \\
2.5 \% \\
\mathrm{NaOCl} \\
2 \% \mathrm{CHX} \\
17 \% \mathrm{EDTA} \\
0.25 \% \mathrm{Ag}- \\
\mathrm{GO}\end{array}$ & Saline & $\begin{array}{l}\text { P. acnes, } \\
\text { A. radicidentis, } \\
\text { S. epidermidis, } \\
\text { and S. mitis }\end{array}$ & $\begin{array}{c}\text { Paper point } \\
\text { sampling/ } \\
\text { microbial counts/ } \\
\text { CLSM }\end{array}$ & $\begin{array}{l}\text { Ag-GO resulted in } \\
\text { significant bacterial } \\
\text { reduction, however, } \\
2.5 \% \mathrm{NaOCl} \\
\text { showed highest } \\
\text { antimicrobial } \\
\text { efficacy }\end{array}$ & $\begin{array}{l}\text { Ioannidis } \\
\text { et al., } \\
2019\end{array}$ \\
\hline
\end{tabular}


from the cell membrane (Al-Jumaili et al., 2017; Zhou and Gao, 2014)

2. Cell entrapment. Aggregated graphene sheets can trap and separate microorganisms from their microenvironment. This environmental disconnection results in preventing nutrient consumption, reducing proliferative activity, and eventually inactivation of the microorganism (Akhavan et al., 2011)

3. Oxidative stress induction can also act as an electron pump transferring electrons out of microorganisms (Liu et al., 2011).

However, the exact antimicrobial effectiveness is difficult to predict and is thought to be dependent on several factors such as type of microorganisms, duration of exposure, and most importantly specific structural properties - such as number of layers, porosity, size, and shape of the individual graphene sheets (Li et al., 2014; Liu et al., 2011; Zhu et al., 2017).

In general, graphene-based materials are biocompatible, promoting cell adhesion, proliferation, and differentiation (Menaa et al., 2015; Tahriri et al., 2019). However, the exact degree of biocompatibility and potential cytotoxicity is highly dependent on their oxygen functional groups (Guazzo et al., 2018).
Despite the limited in vivo studies, in vitro studies of variable methodologies concluded that most graphene-based hydrophilic forms are less toxic than the hydrophobic forms (Guazzo et al., 2018; Tahriri et al., 2019).

To further investigate graphenes' biocompatibility and and dental tissue regeneration, Rosa et al. (2016) investigated the proliferation and differentiation potential of DPSCs on graphene oxide-based substrates. Their results indicated an upregulation of odontogenic gene expression with graphene oxide-based substrates, in comparison to the control (glass-based substrates). The same group later observed higher osteogenic rather than odontoblastic differentiation (Xie et al., 2017). However, in vitro studies do not provide robust data distinction between osteogenic and odontogenic differentiation, and further in vivo studies are required to make this distinction.

More recently, graphene-based materials have gained significant attention within the dental field, including graphene coated implants (Jung et al., 2015; Ren et al., 2017), incorporation into commercial dental materials such as adhesives and resins (Bregnocchi et al., 2017; Sun et al., 2018), and as

Table 1f. In vitro and ex vivo studies of alternative antimicrobial strategies highlighting antimicrobial type, origin, study design, usage, study and control groups, assessment method and duration, microorganisms tested and main findings.

\begin{tabular}{|c|c|c|c|c|c|c|c|}
\hline $\begin{array}{c}\text { Alternative } \\
\text { antimicrobial/ } \\
\text { origin }\end{array}$ & $\begin{array}{l}\text { Study } \\
\text { design/ } \\
\text { usage }\end{array}$ & Study groups & $\begin{array}{l}\text { Control } \\
\text { groups }\end{array}$ & Microorganism & $\begin{array}{c}\text { Assessment } \\
\text { method/ } \\
\text { duration }\end{array}$ & Main findings & Reference \\
\hline $\begin{array}{l}\text { AgNPs in } \\
\text { an aqueous } \\
\text { vehicle/ } \\
\text { Khemia, São } \\
\text { Paulo, Brazil }\end{array}$ & $\begin{array}{l}\text { Bovine } \\
\text { dentine } \\
\text { blocks }\end{array}$ & $\begin{array}{c}94 \text { ppm AgNPs } \\
\text { solution } \\
2.5 \% \mathrm{NaOCl} \\
2 \% \mathrm{CHX}\end{array}$ & $\begin{array}{c}\text { Saline } \\
\text { without } \\
\text { inoculum }\end{array}$ & $\begin{array}{l}\text { E. faecalis } \\
\text { biofilm and } \\
\text { infected } \\
\text { dentinal } \\
\text { tubules }\end{array}$ & $\begin{array}{c}\text { LIVE/ } \\
\text { DEAD } \\
\text { staining } \\
\text { Variable } \\
\text { contact } \\
\text { time of } \\
5,15 \text { and } \\
30 \text { min }\end{array}$ & $\begin{array}{c}\text { AgNPs showed } \\
\text { variable antimicrobial } \\
\text { efficacy; however, } \\
\text { this was inferior to } \\
\mathrm{NaOCl}\end{array}$ & $\begin{array}{l}\text { Rodrigues } \\
\text { et al., } \\
2018\end{array}$ \\
\hline $\begin{array}{l}\text { Bioactive } \\
\text { glass S53P4 }\end{array}$ & $\begin{array}{c}\text { Contra- } \\
\text { lateral } \\
\text { human } \\
\text { premolar } \\
\text { teeth } \\
\text { Medicament }\end{array}$ & $\begin{array}{c}\text { Bioactive glass } \\
\mathrm{Ca}(\mathrm{OH})_{2}\end{array}$ & $\begin{array}{c}\text { No } \\
\text { medicament }\end{array}$ & E. faecalis & $\begin{array}{l}\text { Sampling } \\
\text { of dentine } \\
\text { chips/ } \\
\text { SEM } \\
10 \mathrm{~d}\end{array}$ & $\begin{array}{l}\text { Bioactive glass, in its } \\
\text { current format, was } \\
\text { less efficient than } \\
\text { standard } \mathrm{Ca}(\mathrm{OH})_{2}\end{array}$ & $\begin{array}{l}\text { Zehnder } \\
\text { et al., } \\
2006\end{array}$ \\
\hline $\begin{array}{c}\text { Bioactive } \\
\text { glass S53P4 }\end{array}$ & $\begin{array}{c}\begin{array}{c}\text { Human } \\
\text { dentine } \\
\text { block model }\end{array} \\
\text { Medicament }\end{array}$ & $\begin{array}{c}2 \% \text { CHX gel } \\
2 \% \\
\text { metronidazole } \\
\text { gel } \\
\text { Bioactive glass } \\
\mathrm{Ca}(\mathrm{OH})_{2}\end{array}$ & Saline & E. faecalis & $\begin{array}{c}\text { CFUs/ } \\
\text { inhibition } \\
\text { of growth } \\
1,3 \text { and } 5 \mathrm{~d}\end{array}$ & $\begin{array}{c}\text { Bioactive glass S53P4 } \\
\text { antimicrobial efficacy } \\
\text { was superior to } \\
\mathrm{Ca}(\mathrm{OH})_{2^{\prime}} \text { but inferior } \\
\text { to metronidazole and } \\
\mathrm{CHX} \text { gel }\end{array}$ & $\begin{array}{c}\text { Krithikadatta } \\
\text { et al., } \\
2007\end{array}$ \\
\hline $\begin{array}{c}\text { Bioactive } \\
\text { glass S53P4 }\end{array}$ & $\begin{array}{l}\text { Human root } \\
\text { model } \\
\text { Medicament }\end{array}$ & $\begin{array}{c}\mathrm{Ca}(\mathrm{OH})_{2} \\
1 \% \mathrm{CHX} \text { gel } \\
\text { bioactive glass } \\
\mathrm{Ca}(\mathrm{OH})_{2} \text { plus } \\
\text { point } \\
\text { Activ point }\end{array}$ & Saline & $\begin{array}{l}\text { E. faecalis and } \\
\text { S. mutans }\end{array}$ & $\begin{array}{l}\text { CFUs } \\
7 \mathrm{~d}\end{array}$ & $\begin{array}{l}\mathrm{CHX} \text {-impregnated } \\
\text { medicaments were } \\
\text { more efficient than } \\
\text { alkaline-pH-acting } \\
\text { medicaments }\end{array}$ & $\begin{array}{l}\text { Atila-Pektaş } \\
\text { et al., } \\
2013\end{array}$ \\
\hline $\begin{array}{c}\text { TAMP } \\
\text { bioactive glass }\end{array}$ & $\begin{array}{c}\begin{array}{c}\text { Human } \\
\text { dentine } \\
\text { block model }\end{array} \\
\text { Medicament }\end{array}$ & $\begin{array}{c}\text { TAMP- } \\
\text { bioactive glass } \\
100 \mathrm{mg} / \mathrm{mL} \\
\text { DAP } 1 \mathrm{mg} / \mathrm{mL} \\
0.02 \% \text { AgNPs } \\
\text { gel }\end{array}$ & $\begin{array}{c}\text { Without } \\
\text { bacterial } \\
\text { contamination }\end{array}$ & $\begin{array}{l}\text { E. faecalis } \\
\text { biofilms } \\
\text { and infected } \\
\text { dentinal } \\
\text { tubules }\end{array}$ & $\begin{array}{l}\text { Biofilm } \\
\text { disruption } \\
\text { assay/ } \\
\text { CFUs/SEM } \\
1 \text { and } 7 \mathrm{~d}\end{array}$ & $\begin{array}{c}\text { All tested } \\
\text { medicaments } \\
\text { resulted in significant } \\
\text { biofilms reduction }\end{array}$ & $\begin{array}{l}\text { Sadek } \\
\text { et al., } \\
2019\end{array}$ \\
\hline
\end{tabular}


bioactive cements (Dubey et al., 2017). To enhance root canal disinfection, the synthesis of AgNPs on an aqueous graphene oxide matrix has shown promising preliminary results in terms of antimicrobial activity and biofilm disruption (Ioannidis et al., 2019).

Their versatile and promising properties position graphenes as suitable candidates for investigation within RET. Emerging studies on them as intra-canal disinfectant agents are summarised in Table 1.

\section{Pectin}

Pectin is a natural plant-specific carboxylated polysaccharide extracted from fruits or vegetables. Pectin provides mechanical strength for the cell walls of plants and are important for various cellular processes, such as water absorption, morphological development, and ripening of fruits (RedondoNevado et al., 2001; Vincken et al., 2003). Pectins are known for their gelling, thickening and emulsifying properties, hence their wide use in the food industry. Its emulsification property is affected by the chemical characteristics of the raw material as well as extraction methods used and can be modified using chemical and enzymatic treatments.

The gelling properties of pectin became of interest due to its possible use as an in situ biocompatible gelling system for bone tissue engineering (Munarin et al., 2010a; Munarin et al., 2011; Munarin et al., 2012), an injectable cell delivery system (Mishra et al., 2008), and a drug delivery system (Ishii and Matsunaga, 2001; Munarin et al., 2010b). The structure and features of pectins depend on the plant species and tissues. However, some characteristics are common to all of these polysaccharides. Two main structural categories have been recognised, the smooth and hairy (or ramified) regions (Varoni et al., 2012).

Plant-derived pectins have been investigated as candidates for surface nano-coating of orthopaedic and dental titanium implants, due to their ability to enhance osteogenic differentiation of osteoblasts. Folkert et al. (2016) investigated the effect of coating titanium implants, with unmodified pectin and enzymatically modified pectin extracted from potato pulps, on in vitro cell proliferation, mineralisation, and osteogenic differentiation of osteoblast-like cell lines and primary mice osteoblasts. The study confirmed that both types of pectin coating enhanced cell proliferation, mineralisation, and osteoblastic differentiation - particularly modified pectins with a high content of galactose (Folkert et al., 2016).

Conversely, Gurzawska et al. (2017) evaluated the effect of nano-coating titanium implants with plantcell-wall-derived rhamnogalacturonan-I (pectins), on the bone healing and osseointegration of implants in the tibia of a rabbit model. The results showed no significant difference between coated and non-coated implants.

In dentistry, Nguyen et al. (2013) found that different pectins (LM-, HM- and AM-pectin) coated liposomes, adsorbed the hydroxyapatite to the tooth surfaces in vitro which suggested their possible usage as a protective coating on tooth enamel. Furthermore,

Table 1g. In vitro and ex vivo studies of alternative antimicrobial strategies highlighting antimicrobial type, origin, study design, usage, study and control groups, assessment method and duration, microorganisms tested and main findings.

\begin{tabular}{|c|c|c|c|c|c|c|c|}
\hline $\begin{array}{c}\text { Alternative } \\
\text { antimicrobial/ } \\
\text { origin }\end{array}$ & $\begin{array}{l}\text { Study } \\
\text { design/ } \\
\text { usage }\end{array}$ & Study groups & Control groups & Microorganism & $\begin{array}{c}\text { Assessment } \\
\text { method/ } \\
\text { duration }\end{array}$ & Main findings & Reference \\
\hline $\begin{array}{c}\text { Poly } \\
\text { (lactic- co -glycolic } \\
\text { acid) nanoparticles } \\
\text { with methylene } \\
\text { blue as a } \\
\text { photosensitiser }\end{array}$ & $\begin{array}{l}\text { Human } \\
\text { root } \\
\text { model }\end{array}$ & $\begin{array}{c}\text { Methylene } \\
\text { blue loaded } \\
\text { nanoparticles } \\
\text { with/without } \\
\text { light }\end{array}$ & $\begin{array}{l}\text { No light/ } \\
\text { no methylene } \\
\text { blue } \\
\text { nanoparticles }\end{array}$ & $\begin{array}{l}\text { E. faecalis in } \\
\text { planktonic and } \\
\text { biofilm forms }\end{array}$ & $\begin{array}{l}\text { CFUs/ SEM } \\
\text { Light } \\
\text { application } \\
\text { for } 5 \text { min } \\
\text { with a } \\
\text { wavelength } \\
\text { of } 665 \mathrm{~nm} \text {. }\end{array}$ & $\begin{array}{c}\text { Methylene } \\
\text { blue loaded } \\
\text { nanoparticles } \\
\text { reduced E. faecalis } \\
\text { counts in both } \\
\text { planktonic phase } \\
\text { and within root } \\
\text { canals }\end{array}$ & $\begin{array}{l}\text { Pagonis } \\
\text { et al., } \\
2010\end{array}$ \\
\hline $\begin{array}{c}\text { Curcumin/ } \\
\text { indocyanine } \\
\text { green as } \\
\text { photosensitiser }\end{array}$ & $\begin{array}{c}\text { Well } \\
\text { plates } \\
\text { Irrigant }\end{array}$ & $\begin{array}{c}5.25 \% \\
\mathrm{NaOCl}, \\
0.2 \% \mathrm{CHX}, \\
2 \% \mathrm{CHX}, \\
40 \mathrm{mmol} / \mathrm{L} \\
\text { curcumin } \\
\text { with LED } \\
1 \mathrm{mg} / \mathrm{mL} \\
\text { indocyanine } \\
\text { green with } \\
\text { diode laser }\end{array}$ & $\begin{array}{c}\text { No exposure } \\
\text { to irrigation } \\
\text { solutions or } \\
\text { photosensitisers }\end{array}$ & $\begin{array}{c}\text { E. faecalis in } \\
\text { planktonic and } \\
\text { biofilm forms }\end{array}$ & $\begin{array}{c}\text { CFUs/ } \\
\text { crystal violet } \\
\text { assay } \\
\text { LED } \\
\text { wavelength } \\
\text { of } 450 \mathrm{~nm} \\
\text { Diode laser } \\
\text { wavelength } \\
\text { of } 810 \mathrm{~nm} \\
\end{array}$ & $\begin{array}{l}\text { Photoactivated } \\
\text { curcumin } \\
\text { antimicrobial } \\
\text { efficacy was very } \\
\text { close to standard } \\
\text { irrigating solutions } \\
\text { tested }\end{array}$ & $\begin{array}{c}\text { Pourhajibagher } \\
\text { et al., } \\
2018\end{array}$ \\
\hline $\begin{array}{c}\text { rGO-Cur } \\
\text { photosensitiser }\end{array}$ & $\begin{array}{l}\text { Human } \\
\text { root } \\
\text { model }\end{array}$ & $\begin{array}{l}\text { rGO-Cur } \\
\text { LED group } \\
\text { rGO-Cur } \\
\text { with LED } \\
2.5 \% \mathrm{NaOCl}\end{array}$ & $\begin{array}{l}\text { Only bacterial } \\
\text { suspension }\end{array}$ & $\begin{array}{l}\text { E. faecalis } \\
\text { biofilms }\end{array}$ & $\begin{array}{l}\text { MIC/ SEM/ } \\
\text { real-time } \\
\text { qPCR } \\
\text { Wavelength } \\
\text { of } \\
450 \pm 30 \mathrm{~nm} \\
\text { with } 300 \mathrm{~s}\end{array}$ & $\begin{array}{l}\text { The combined } \\
\text { usage of rGO- } \\
\text { Cur with LED } \\
\text { showed promising } \\
\text { results in terms } \\
\text { of antimicrobial } \\
\text { efficacy and biofilm } \\
\text { inhibition }\end{array}$ & $\begin{array}{c}\text { Ghorbanzadeh } \\
\text { et al., } \\
2020\end{array}$ \\
\hline
\end{tabular}


in vivo usage of pectin in combination with a chitosan scaffold within RET in dog endodontic lesions was assessed (Palma et al., 2017). However, the mineral content and root closure were better using a blood clot alone, in comparison to a pectin-chitosan scaffold (Palma et al., 2017).

Pectin is reported to have bactericidal effects against the most widely distributed pathogenic and opportunistic microorganisms. It was also found that higher concentrations ( $>2 \%$ ) had an inactivating effect on a therapeutic bacteriophage and decreased the antimicrobial activity of penicillin (Men'shikov et al., 1997). Cinnamaldehyde pectin extracted from the papaya puree were found to have antimicrobial effects against E. coli, L. monocytogenes, S. aureus, and S. enterica (Otoni et al., 2014).

Although it is still early days for pectin usage in the dental field, its gelling properties, unique structural variations, and antimicrobial properties, makes injectable pectin gels an excellent candidate to investigate for regenerative endodontics.

\section{Ascorbic acid}

Ascorbic acid, commonly known as vitamin C, is an essential vitamin for the health of body tissues and especially for natural collagen synthesis. This white to light-yellow water-soluble ketolactone with two ionisable hydroxyl groups (Du et al., 2012), is the most abundant antioxidant vitamin within plant cells (Smirnoff, 2000).

As an electron-donor, ascorbic acid is known to have several physiological and biochemical properties (Arrigoni and De Tullio, 2002; Du et al.,
2012). Ascorbic acid has antimicrobial properties against several bacteria and viruses, ranging from bacteriostatic to bactericidal that was reported to increase by time (Goldschmidt, 1991).

Ascorbic acid also has potent anti-inflammatory properties (Diomede et al., 2020), therefore; positively influencing host-defence mechanism towards tissues repair during infection (Goldschmidt, 1991). Its antiscorbutic properties play a pivotal role in the synthesis of collagen through its ability to act as an electron-donor maintaining the ferrous in collagen hydroxylases in an active state (Du et al., 2012). Ascorbic acid's potential influence on the formation of dentine collagen matrix is of particular interest to researchers in pulp-dentine complex regeneration (Balic et al., 2009).

Within the dental field, ascorbic acid has been advocated as a biocompatible reducing agent integrated into commercial monomers and composite systems. Indeed, $10 \%$ ascorbic acid was found to restore the dentine bonding strength to adhesive resins after deproteinisation with $5 \% \mathrm{NaOCl}$ irrigation solution (da Cunha et al., 2010; Furuse et al., 2014; Morris et al., 2001). Additionally, the integration of ascorbic acid within dental monomer increased DPSCs proliferation rate and decreased unwanted cellular morphology alterations due to the monomer treatment (Diomede et al., 2020).

Furthermore, its potential role in promoting regeneration of the dental pulp, as a novel endodontic compound, has been suggested (Diomede et al., 2020). Its relative cheap cost, ease of use, antioxidant properties, and tissue repair properties makes it an

Table 2. In vivo human and animal studies of alternatives antimicrobial strategies highlighting antimicrobial type, origin, study design, usage, study and control groups, assessment method and duration and main findings.

\begin{tabular}{|c|c|c|c|c|c|c|}
\hline $\begin{array}{c}\text { Material/ } \\
\text { origin/ } \\
\text { preparation }\end{array}$ & $\begin{array}{c}\text { Study design/ } \\
\text { usage }\end{array}$ & Study groups & $\begin{array}{l}\text { Control } \\
\text { groups }\end{array}$ & $\begin{array}{c}\text { Assessment method/ } \\
\text { duration }\end{array}$ & Main findings & Reference \\
\hline $\begin{array}{c}\text { EEP/ } \\
\text { Commercially } \\
\text { available }\end{array}$ & $\begin{array}{l}\text { Chronically } \\
\text { exposed } \\
\text { primary teeth } \\
\text { Irrigant }\end{array}$ & $\begin{array}{c}3 \% \mathrm{NaOCl} \\
12.5 \% \\
\text { alcoholic } \\
\text { extract of } \\
\text { miswak } \\
11 \% \text { EEP }\end{array}$ & $0.9 \%$ saline & $\begin{array}{l}\text { Paper points sampling/ } \\
\text { CFUs } \\
\text { Pre- and post-irrigation } \\
\text { samples }\end{array}$ & $\begin{array}{c}11 \% \text { EEP showed } \\
\text { disappointing results } \\
\text { with no significance } \\
\text { difference from the } \\
\text { control }\end{array}$ & $\begin{array}{c}\text { Shingare } \\
\text { and } \\
\text { Chaugule, } \\
2011\end{array}$ \\
\hline $\begin{array}{l}\text { Egyptian } \\
\text { propolis/ } \\
\text { El Monofia } \\
\text { province }\end{array}$ & $\begin{array}{l}\text { Immature } \\
\text { non-vital } \\
\text { dogs' teeth } \\
\text { with induced } \\
\text { periapical } \\
\text { infection } \\
\text { Medicament } \\
\text { for RET }\end{array}$ & $\begin{array}{c}\text { Propolis paste } \\
\text { with propolis } \\
\text { plug } \\
\text { Propolis paste } \\
\text { with MTA plug } \\
\text { TAP with } \\
\text { propolis plug } \\
\text { TAP with MTA } \\
\text { plug }\end{array}$ & $\begin{array}{c}\text { No } \\
\text { medicament } \\
\text { No } \\
\text { intervention }\end{array}$ & $\begin{array}{l}\text { Antimicrobial efficacy: } \\
\text { CFUs/reduction in colony } \\
\text { counts } \\
\text { 3 weeks } \\
\text { Regenerative outcome: } \\
\text { radiographic/ } \\
\text { histopathologic evaluation } \\
2 \text { weeks, } 1 \text { and } 2 \text { months }\end{array}$ & $\begin{array}{l}\text { Propolis showed } \\
\text { promising results, } \\
\text { comparable to TAP, in } \\
\text { terms of antimicrobial } \\
\text { efficacy and hard and } \\
\text { soft tissue deposition }\end{array}$ & $\begin{array}{l}\text { El-Tayeb } \\
\text { et al., } \\
2019\end{array}$ \\
\hline $\begin{array}{c}\text { Propolis/ } \\
\text { Farmácia de } \\
\text { Manipulação } \\
\text { Nova Derme }\end{array}$ & $\begin{array}{l}\text { Immature } \\
\text { dog teeth } \\
\text { with induced } \\
\text { periapical } \\
\text { infection } \\
\text { Medicament } \\
\text { for RET }\end{array}$ & $\begin{array}{c}\text { TAP } \\
1 \% \text { propolis } \\
\text { paste }\end{array}$ & $\begin{array}{c}\text { No } \\
\text { medicament } \\
\text { No } \\
\text { intervention }\end{array}$ & $\begin{array}{l}\text { Histological analysis } \\
\qquad 7 \text { months }\end{array}$ & $\begin{array}{c}\text { Propolis showed } \\
\text { promising results, } \\
\text { superior to TAP, in terms } \\
\text { of hard and soft tissue } \\
\text { deposition }\end{array}$ & $\begin{array}{c}\text { Pagliarin } \\
\text { et al., } \\
2016\end{array}$ \\
\hline
\end{tabular}


excellent candidate to investigate for regenerative endodontics.

\section{Synthetic materials and strategies}

\section{Bioactive glass}

In the early 90s, Hench and colleagues developed a novel Class A bioactive glass-ceramic material composed of silica and other components such as calcium and phosphate (Hench and Paschall, 1973; Hench et al., 1971). Following this discovery, bioactive glass (calcium sodium phosphosilicate) received considerable clinical interest within the dental field as a versatile material -as a bone substitute for tissue regeneration (Norton and Wilson, 2002; Pereira et al., 2018), an implant coating material (Xuereb et al., 2015), a drug delivery material (Wu and Chang, 2014), and an additional component within various restorative materials (Sauro et al., 2012; Tirapelli et al., 2011). This attracted research attention was largely related to its inherent material advantages such as antimicrobial activity (Munukka et al., 2008; Waltimo et al., 2007; Zhang et al., 2010), biocompatibility, and hard tissue regenerative potential (El-Gendy et al., 2015; El-Gendy et al., 2013; El Shazley et al., 2016).

The mechanism of antimicrobial action of bioactive glass is attributed to different factors - its alkaline $\mathrm{pH}$, the sustained release of silica and/or calcium phosphate ions, and specific glass composition (Gubler et al., 2008; Zhang et al., 2010). However, because conventional micron-sized bioactive glass demonstrated mild to moderate antimicrobial activity, specifically against $E$. faecalis, material improvements were consistently ongoing (Krithikadatta et al., 2007; Waltimo et al., 2007; Zehnder et al., 2006).

Advances with nano-technology fabrication led to the development of nano-scale bioactive glass (Lei et al., 2010). The nano-scale bioactive glass $45 S 5$ was found to increase the $\mathrm{pH}$ in a solution and increase silica release by a factor of 10 in comparison to $\mu \mathrm{m}$-sized bioactive glass, resulting in improved antimicrobial effectiveness (Waltimo et al., 2007). Increased ion release of nano-scale bioactive glass also enhanced cytocompatibility and tissue regeneration properties (Lei et al., 2010; Wang et al., 2020); nano-scale bioactive glass also promoted dentine mineralisation of higher stability and acid resistance compared to micron-scale glass particles (Sheng et al., 2016).

Various types of bioactive glass have been specifically developed and investigated such as TAMP bioactive glass (Sadek et al., 2019) and mesoporous bioactive glass (Wu et al., 2011; Yan et al., 2004). Furthermore, with improved material science, the ability to customise bioactive glass functionalised structures with the addition of antimicrobial and regenerative agents became possible (Ribeiro et al., 2020). The combined addition of silver ions to mesoporous bioactive glass has shown promising results in terms of improved antimicrobial effectiveness (Gargiulo et al., 2013). More sophisticated hybrid systems have also been developed, such as the incorporation of silver-doped bioactive glass within hydrogels with promising antimicrobial, anti-inflammatory, and DPSCs differentiation potential (Wang et al., 2015; Zhu et al., 2019).

Although at an early stage of research, studies expanding its use as a disinfectant agent for endodontics (Atila-Pektaş et al., 2013; Krithikadatta et al., 2007; Zehnder et al., 2006) and RET (Sadek et al., 2019) have been performed and summarised in Table 1.

\section{PDT}

PDT, also known as photodynamic inactivation or photoactivated disinfection, is advocated as an adjunct antimicrobial approach for clinical disinfection of the complex root canal system (Gursoy et al., 2013; Plotino et al., 2019). This specialised technique involves the vibrant interaction between a photosensitising agent (photosensitiser) and a light source (Hamblin and Hasan, 2004; Konopka and Goslinski, 2007). This interaction leads to the production of reactive oxygen species, such as free radicals and singlet oxygen, resulting in oxidative and cytotoxic damage to the target cells (Hamblin and Hasan, 2004; Konopka and Goslinski, 2007).

The antimicrobial mechanism of PDT can be explained based on the direct effect on extracellular molecules mediated by singlet oxygen of high chemical reactivity and the indirect photodamage to the polysaccharide bacterial biofilm (Konopka and Goslinski, 2007; Wainwright and Crossley, 2004). This dual activity is reported as a significant advantage over currently used antibiotics (Konopka and Goslinski, 2007; Plotino et al., 2019), with effectiveness against antibiotic-sensitive and antibiotic-resistant microorganisms (Wainwright and Crossley, 2004). Furthermore, there is no evidence of bacterial resistance to the various metabolic pathways associated with the action of singlet oxygen or free radicals (Dias et al., 2020; Konopka and Goslinski, 2007).

The effect of PDT on the surrounding stem cells has been investigated. Li et al. (2020) found that the application of PDT provided an inductive microenvironment for SCAP growth. Quantitative reverse transcriptase-polymerase chain reaction also resulted in a positive expression of platelet-derived growth factor and vascular endothelial growth factor. In-line with the above, PDT resulted in greater viability of apical papilla cells (Deluca et al., 2021) and significantly less cytotoxicity compared to $\mathrm{NaOCl}$ irrigation (George and Kishen, 2007; Gomes-Filho et al., 2016).

Within dentistry, the application of PDT is expanding into different areas, such as treatment of head and neck cancer (Grant et al., 1993; Hopper, 2000), oral plaque biofilms (Tahmassebi et al., 2015; Wood et al., 2006), treatment of peri-implantitis 
(Bassetti et al., 2014; Bombeccari et al., 2013), and root canal disinfection (Asnaashari et al., 2017; Bonsor et al., 2006; de Miranda and Colombo, 2018; Soukos et al., 2006). More recently, PDT has been recommended as a positive adjunct in RET protocols (Deluca et al., 2021; Devaraj et al., 2016). However, to date, this is scarcely documented in vivo. Successful clinical and radiographic outcomes, in terms of thickening of dentinal walls and apical closure, following the adjunct use of tolonium chloride photosensitiser followed by platelet-rich fibrin (Johns et al., 2014) or collagen resorbable matrix (Abdel Hafiz Abdel Rahim et al., 2019) are reported.

Although most reported studies utilised PDT with aid of an intra-canal optic fibre, Nunes et al. showed no significant difference in bacterial reduction when an intra-canal optic fibre was not used. However, in this in vitro study, all teeth were decoronated and only standard $15 \mathrm{~mm}$ root segments were utilised (Nunes et al., 2011). The reduced oxygen concentration within the root canals, especially in deep irregularities, can directly affect the formation of cytotoxic oxygen derivatives and reduce the antimicrobial efficacy (Nunes et al., 2011). Modifying the optical fibre tip to improve the clinical performance has been recommended (George and Walsh, 2011).

Chemical phenothiazine dyes such as methylene blue and toluidine blue (tolonium chloride) are often reported within endodontic protocols (Gursoy et al., 2013; Siddiqui et al., 2013). However, potential adverse effects, such as staining and discoloration are documented (Plotino et al., 2019; Ramalho et al., 2017). To overcome this clinical limitation, attempts such as evaluating a therapeutic efficacy window of the chemical dyes have been tested (Gursoy et al., 2013; Plotino et al., 2019). Obliteration of dentinal tubules as a result of viscous photosensitiser substances impregnating the dentine surface is also reported (Plotino et al., 2019). Clinically, this could reduce the bond strength between the root filling material and dentinal walls (Shahravan et al., 2007).

Therefore, to overcome the above limitations and enhance clinical outcomes, research has recently focused on the development of novel formulations, such as polymer-based nanoparticle photosensitiser (Gil-Tomás et al., 2007; Koo et al., 2007; Shrestha and Kishen, 2014). Nanoparticlebased photosensitisers have several advantages over standard photosensitising molecules such as:

1. increasing production of reactive oxygen species;

2. reducing the possibility of multiple-drug resistance;

3. providing selective treatment by localised delivery agents;

4. having a nonimmunogenic nature of nanoparticle matrix (Koo et al., 2007; Pagonis et al., 2010).

Examples of novel photosensitiser suggested for intra-canal disinfection include poly(lacticcoglycolic) acid nanoparticles loaded with methylene blue (Pagonis et al., 2010). The use of curcumin solution as a photosensitiser has gained significant scientific interest (da Frota et al., 2015; Ghorbanzadeh et al., 2020; Neelakantan et al., 2015; Pourhajibagher et al., 2018; Sotomil et al., 2019). Chitosan nanoparticles functionalised with rose-bengal photosensitiser were also found to stabilise the structural integrity of root dentine in vitro by photo-crosslinking the collagen, resulting in sufficient elimination of biofilms, the stabilisation of the dentinal matrix (Shrestha and Kishen, 2014), and significant inactivation of bacterial endotoxin lipopolysaccharides (Shrestha et al., 2015). Emerging studies are currently being published of a novel photosensitiser as an adjunct for intra-canal disinfection, with promising results, as summarised in Table 1.

\section{Concluding remarks and future perspectives}

Adequate disinfection of the root canal system during RET is a prerequisite for successful regeneration of the pulp-dentinal complex. However, it should be achieved while maintaining a conducive environment for stem cell survival and proliferation. Although currently adopted antimicrobial protocols provide acceptable disinfection, the clinical outcomes are still unpredictable and far from ideal. Key limitations, such as coronal discolouration, cell cytotoxicity, difficulty of removal from the root canal, development of sensitisation and resistant bacterial strains are widely documented within the literature.

There is a growing interest in the exploration of alternative antimicrobial strategies within RET for a predictable biological outcome. Despite the above-mentioned promising results of various new strategies, it is noteworthy that currently available data are mostly drawn from in vitro and limited animal studies using single bacterial species, mainly E. faecalis. Further investigations into the effect of the proposed antimicrobials against complex polymicrobial biofilms involved in endodontic infections is of extreme importance to finalise the conclusion concerning the use of these materials in RET. Therefore, the development and testing of the proposed alternative antimicrobial materials within a well-controlled in vitro, followed by in vivo, studies are required.

\section{Acknowledgements}

All authors confirm no conflicts of interest with any organisation regarding the materials discussed in the review.

\section{References}

Adamczak A, Ożarowski M, Karpiński TM (2020) Curcumin, a natural antimicrobial agent with strainspecific activity. Pharmaceuticals 13: 1-12. 
Afkhami F, Elahy S, Mahmoudi-Nahavandi A (2017) Spectrophotometric analysis of crown discoloration following the use of silver nanoparticles combined with calcium hydroxide as intracanal medicament. J Clin Exp Dent 9: e842-e847.

Afkhami F, Pourhashemi SJ, Sadegh M, Salehi Y, Fard MJK (2015) Antibiofilm efficacy of silver nanoparticles as a vehicle for calcium hydroxide medicament against Enterococcus faecalis. J Dent 43: 1573-1579.

Agnihotri SA, Mallikarjuna NN, Aminabhavi TM (2004) Recent advances on chitosan-based micro- and nanoparticles in drug delivery. J Control Release 100: 5-28.

Ahangari Z, Naseri M, Jalili M, Mansouri Y, Mashhadiabbas F, Torkaman A (2012) Effect of propolis on dentin regeneration and the potential role of dental pulp stem cell in guinea pigs. Cell J 13: 223-228.

Akhavan O, Ghaderi E, Esfandiar A (2011) Wrapping bacteria by graphene nanosheets for isolation from environment, reactivation by sonication, and inactivation by near-infrared irradiation. J Phys Chem B 115: 6279-6288.

Al-Jumaili A, Alancherry S, Bazaka K, Jacob MV (2017) Review on the antimicrobial properties of carbon nanostructures. Materials (Basel) 10: 1066. DOI: $10.3390 / \mathrm{ma} 10091066$.

Al-Shaher A, Wallace J, Agarwal S, Bretz W, Baugh D (2004) Effect of propolis on human fibroblasts from the pulp and periodontal ligament. J Endod 30: 359361.

Alt V, Bechert T, Steinrücke P, Wagener M, Seidel P, Dingeldein E, Domann E, Schnettler R (2004) An in vitro assessment of the antibacterial properties and cytotoxicity of nanoparticulate silver bone cement. Biomaterials 25: 4383-4391.

Althumairy RI, Teixeira FB, Diogenes A (2014) Effect of dentin conditioning with intracanal medicaments on survival of stem cells of apical papilla. J Endod 40: 521-525.

Arrigoni O, De Tullio MC (2002) Ascorbic acid: much more than just an antioxidant. Biochim Biophys Acta 1569: 1-9.

Asnaashari M, Ashraf H, Rahmati A, Amini N (2017) A comparison between effect of photodynamic therapy by LED and calcium hydroxide therapy for root canal disinfection against Enterococcus faecalis: a randomized controlled trial. Photodiagn Photodyn Ther 17: 226-232.

Atila-Pektaş B, Yurdakul P, Gülmez D, Görduysus Ö (2013) Antimicrobial effects of root canal medicaments against Enterococcus faecalis and Streptococcus mutans. Int Endod J 46: 413-418.

Ayoub S, Cheayto A, Bassam S, Najar M, Berbéri A, Fayyad-Kazan M (2020) The effects of intracanal irrigants and medicaments on dental-derived stem cells fate in regenerative endodontics: an update. Stem Cell Rev Rep 16: 650-660.

Balic A, Rodgers B, Mina M (2009) Mineralization and expression of Col1a1-3.6GFP transgene in primary dental pulp culture. Cells Tissues Organs 189: 163-168.

Ballal NV, Shavi GV, Kumar R, Kundabala M, Bhat KS (2010) In vitro sustained release of calcium ions and ph maintenance from different vehicles containing calcium hydroxide. J Endod 36: 862-866.

Banchs F, Trope M (2004) Revascularization of immature permanent teeth with apical periodontitis: new treatment protocol? J Endod 30: 196-200.

Bapat RA, Chaubal TV, Joshi CP, Bapat PR, Choudhury H, Pandey M, Gorain B, Kesharwani P (2018) An overview of application of silver nanoparticles for biomaterials in dentistry. Mater Sci Eng C 91: 881-898.

Baranwal R, Duggi V, Avinash A, Dubey A, Pagaria S, Munot H (2017) Propolis: a smart supplement for an intracanal medicament. Int J Clin Pediatr Dent 10: 324-329.

Bassetti M, Schär D, Wicki B, Eick S, Ramseier CA, Arweiler NB, Sculean A, Salvi GE (2014) Antiinfective therapy of peri-implantitis with adjunctive local drug delivery or photodynamic therapy: 12-month outcomes of a randomized controlled clinical trial. Clin Oral Implants Res 25: 279-287.

Berretta AA, Nascimento AP, Bueno PC, Vaz MM, Marchetti JM (2012) Propolis standardized extract $\left(\mathrm{EPP}-\mathrm{AF}^{\circledR}\right)$, an innovative chemically and biologically reproducible pharmaceutical compound for treating wounds. Int J Biol Sci 8: 512-521.

Berkhoff JA, Chen PB, Teixeira FB, Diogenes A (2014) Evaluation of triple antibiotic paste removal by different irrigation procedures. J Endod 40: 11721177.

Bombeccari GP, Guzzi G, Gualini F, Gualini S, Santoro F, Spadari F (2013) Photodynamic therapy to treat periimplantitis. Implant Dent 22: 631-638.

Bonsor SJ, Nichol R, Reid TMS, Pearson GJ (2006) An alternative regimen for root canal disinfection. $\mathrm{Br}$ Dent J 201: 101-105.

Bregnocchi A, Zanni E, Uccelletti D, Marra F, Cavallini D, De Angelis F, De Bellis G, Bossù M, Ierardo G, Polimeni A, Sarto MS (2017) Graphenebased dental adhesive with anti-biofilm activity. J Nanobiotechnology 15: 89. DOI: 10.1186/s12951-0170322-1.

Bryce G, O'Donnell D, Ready D, Ng Y-l, Pratten J, Gulabivala K (2009) Contemporary root canal irrigants are able to disrupt and eradicate single- and dual-species biofilms. J Endod 35: 1243-1248.

Cao H, Liu X, Meng F, Chu PK (2011) Biological actions of silver nanoparticles embedded in titanium controlled by micro-galvanic effects. Biomaterials 32: 693-705.

Casagrande L, Demarco FF, Zhang Z, Araujo FB, Shi S, Nör JE (2010) Dentin-derived BMP-2 and odontoblast differentiation. J Dent Res 89: 603-608.

Chang M, Wu M, Li H (2018) Antitumor activities of novel glycyrrhetinic acid-modified curcuminloaded cationic liposomes in vitro and in H22 tumorbearing mice. Drug Deliv 25: 1984-1995. 
Cheng L, Weir MD, Xu HHK, Antonucci JM, Kraigsley AM, Lin NJ, Lin-Gibson S, Zhou X (2012) Antibacterial amorphous calcium phosphate nanocomposites with a quaternary ammonium dimethacrylate and silver nanoparticles. Dent Mater 28: 561-572.

Chung SH, Park Y-S (2017) Local drug delivery in endodontics: a literature review. J Drug Deliv Sci Technol 39: 334-340.

Conde MC, Chisini LA, Demarco FF, Nör JE, Casagrande L, Tarquinio SB (2016) Stem cellbased pulp tissue engineering: variables enrolled in translation from the bench to the bedside, a systematic review of literature. Int Endod J 49: 543550.

Cunha IBS, Sawaya ACHF, Caetano FM, Shimizu MT, Marcucci MC, Drezza FT, Povia GS, Carvalho PDO (2004) Factors that influence the yield and composition of Brazilian propolis extracts. J Braz Chem Soc 15: 964-970.

da Cunha LF, Furuse AY, Mondelli RF, Mondelli J (2010) Compromised bond strength after root dentin deproteinization reversed with ascorbic acid. J Endod 36: 130-134.

da Frota MF, Guerreiro-Tanomaru JM, TanomaruFilho M, Bagnato VS, Espir CG, Berbert FLCV (2015) Photodynamic therapy in root canals contaminated with Enterococcus faecalis using curcumin as photosensitizer. Lasers Med Sci 30: 1867-1872.

de-Jesus-Soares A, Prado MC, Nardello LCL, Pereira AC, Cerqueira-Neto ACCL, Nagata JY, Martinez EF, Frozoni M, Gomes BPFA, Pinheiro ET (2020) Clinical and molecular microbiological evaluation of regenerative endodontic procedures in immature permanent teeth. J Endod 46: 1448-1454.

de Miranda RG, Colombo APV (2018) Clinical and microbiological effectiveness of photodynamic therapy on primary endodontic infections: a 6-month randomized clinical trial. Clin Oral Investig 22: 17511761.

del Carpio-Perochena A, Kishen A, Felitti R, Bhagirath AY, Medapati MR, Lai C, Cunha RS (2017) Antibacterial properties of chitosan nanoparticles and propolis associated with calcium hydroxide against single- and multispecies biofilms: an in vitro and in situ study. J Endod 43: 1332-1336.

Deluca MCdC, Scarparo RK, Aspesi M, Matte BF, Brand LM, Grecca FS, Casagrande L, Kopper PMP (2021) Cytotoxic, migration, and angiogenic effects of photodynamic therapy and photobiomodulation associated with a revascularization protocol. J Endod 47: 69-77.

Devaraj S, Jagannathan N, Neelakantan P (2016) Antibiofilm efficacy of photoactivated curcumin, triple and double antibiotic paste, $2 \%$ chlorhexidine and calcium hydroxide against Enterococcus fecalis in vitro. Sci Rep 6: 24797.

Dias LD, Blanco KC, Mfouo-Tynga IS, Inada NM, Bagnato VS (2020) Curcumin as a photosensitizer: from molecular structure to recent advances in antimicrobial photodynamic therapy. J
Photochem Photobiol C 45: 100384. DOI: 10.1016/j. jphotochemrev.2020.100384.

Diogenes AR, Ruparel NB, Teixeira FB, Hargreaves KM (2014) Translational science in disinfection for regenerative endodontics. J Endod 40: S52-57.

Diomede F, Marconi GD, Guarnieri S, D'Attilio M, Cavalcanti MFXB, Mariggiò MA, Pizzicannella J, Trubiani O (2020) A novel role of ascorbic acid in anti-inflammatory pathway and ROS generation in HEMA treated dental pulp stem cells. Materials 13: 130. DOI: $10.3390 / \mathrm{ma13010130.}$

Dodwad V, Kukreja BJ (2011) Propolis mouthwash: a new beginning. J Indian Soc Periodontol 15: 121-125.

Dotto L, Sarkis Onofre R, Bacchi A, Rocha Pereira GK (2020) Effect of root canal irrigants on the mechanical properties of endodontically treated teeth: a scoping review. J Endod 46: 596-604.e593.

Drake PL, Hazelwood KJ (2005) Exposure-related health effects of silver and silver compounds: a review. Ann Occup Hyg 49: 575-585.

Du J, Cullen JJ, Buettner GR (2012) Ascorbic acid: chemistry, biology and the treatment of cancer. Biochim Biophys Acta 1826: 443-457.

Dubey N, Rajan SS, Bello YD, Min KS, Rosa V (2017) Graphene nanosheets to improve physicomechanical properties of bioactive calcium silicate cements. Materials (Basel) 10: 606. DOI: 10.3390/ ma10060606.

Ducret M, Montembault A, Josse J, Pasdeloup M, Celle A, Benchrih R, Mallein-Gerin F, AlliotLicht B, David L, Farges J-C (2019) Design and characterization of a chitosan-enriched fibrin hydrogel for human dental pulp regeneration. Dent Mater 35: 523-533.

Durner J, Stojanovic M, Urcan E, Hickel R, Reichl F-X (2011) Influence of silver nano-particles on monomer elution from light-cured composites. Dent Mater 27: 631-636.

Dutta AK, Ikiki E (2013) Novel drug delivery systems to improve bioavailability of curcumin. J Bioequiv Bioavailab 6: 1-9.

El-Gendy R, Kirkham J, Newby PJ, Mohanram Y, Boccaccini AR, Yang XB (2015) Investigating the vascularization of tissue-engineered bone constructs using dental pulp cells and 45S5 Bioglass ${ }^{\circ}$ scaffolds. Tissue Eng Part A 21: 2034-2043.

El-Gendy R, Yang XB, Newby PJ, Boccaccini AR, Kirkham J (2013) Osteogenic differentiation of human dental pulp stromal cells on 4555 Bioglass ${ }^{\circledR}$ based scaffolds in vitro and in vivo. Tissue Eng Part A 19: 707-715.

El-Tayeb MM, Abu-Seida AM, El Ashry SH, ElHady SA (2019) Evaluation of antibacterial activity of propolis on regenerative potential of necrotic immature permanent teeth in dogs. BMC Oral Health 19: 174. DOI: 10.1186/s12903-019-0835-0.

El Shazley N, Hamdy A, El-Eneen HA, El Backly RM, Saad MM, Essam W, Moussa H, El Tantawi M, Jain H, Marei MK (2016) Bioglass in alveolar bone regeneration in orthodontic patients: randomized controlled clinical trial. JDR Clin Trans Res 1: 244-255. 
Fan W, Wu D, Tay FR, Ma T, Wu Y, Fan B (2014) Effects of adsorbed and templated nanosilver in mesoporous calcium-silicate nanoparticles on inhibition of bacteria colonization of dentin. Int J Nanomedicine 9: 5217-5230.

Fayaz AM, Balaji K, Girilal M, Yadav R, Kalaichelvan PT, Venketesan R (2010) Biogenic synthesis of silver nanoparticles and their synergistic effect with antibiotics: a study against gram-positive and gram-negative bacteria. Nanomedicine: NBM 6: 103-109.

Folkert J, Meresta A, Gaber T, Miksch K, Buttgereit F, Detert J, Pischon N, Gurzawska K (2016) Nanocoating with plant-derived pectins activates osteoblast response in vitro. Int J Nanomedicine 12: 239-249.

Fouad AF (2020) Contemporary microbial and antimicrobial considerations in regenerative endodontic therapy. J Endod 46: S105-S114.

Franková J, Pivodová V, Vágnerová $H$, Juráňová J, Ulrichová J (2016) Effects of silver nanoparticles on primary cell cultures of fibroblasts and keratinocytes in a wound-healing model. J Appl Biomater Funct 14: 137-142.

Furuse AY, Cunha LF, Baratto SP, Leonardi DP, Haragushiku GA, Gonzaga CC (2014) Bond strength of fiber-reinforced posts to deproteinized root canal dentin. J Contemp Dent Pract 15: 581-586.

Galler KM (2016) Clinical procedures for revitalization: current knowledge and considerations. Int Endod J 49: 926-936.

Galler KM, D'Souza RN, Federlin M, Cavender AC, Hartgerink JD, Hecker S, Schmalz G (2011) Dentin conditioning codetermines cell fate in regenerative endodontics. J Endod 37: 1536-1541.

Galler KM, Krastl G, Simon S, Van Gorp G, Meschi N, Vahedi B, Lambrechts P (2016) European society of endodontology position statement: revitalization procedures. Int Endod J 49: 717-723.

Garcia-Godoy F, Murray PE (2012) Recommendations for using regenerative endodontic procedures in permanent immature traumatized teeth. Dent Traumatol 28: 33-41.

Gargiulo N, Cusano AM, Causa F, Caputo D, Netti PA (2013) Silver-containing mesoporous bioactive glass with improved antibacterial properties. J Mater Sci Mater Med 24: 2129-2135.

George R, Walsh LJ (2011) Performance assessment of novel side firing safe tips for endodontic applications. J Biomed Opt 16: 048004.

George S, Kishen A (2007) Advanced noninvasive light-activated disinfection: assessment of cytotoxicity on fibroblast versus antimicrobial activity against enterococcus faecalis. J Endod 33: 599-602.

Ghisalberti EL (1979) Propolis: a review. Bee World 60: 59-84.

Ghorbanzadeh R, Assadian H, Chiniforush N, ParkerS, Pourakbari B, Ehsani B, Alikhani MY, Bahador A (2020) Modulation of virulence in enterococcus faecalis cells surviving antimicrobial photodynamic inactivation with reduced graphene oxide-curcumin: an ex vivo biofilm model. Photodiagn Photodyn Ther 29: 101643. DOI: 10.1016/j.pdpdt.2019.101643.

Gil-Tomás J, Tubby S, Parkin IP, Narband N, Dekker L, Nair SP, Wilson M, Street C (2007) Lethal photosensitisation of Staphylococcus aureus using a toluidine blue O-tiopronin-gold nanoparticle conjugate. J Mater Chem 17: 3739-3746.

Goldschmidt MC (1991) Reduced bactericidal activity in neutrophils from scorbutic animals and the effect of ascorbic acid on these target bacteria in vivo and in vitro. Am J Clin Nutr 54: 1214S-1220S.

Gomes-Filho JE, Silva FO, Watanabe S, Angelo Cintra LT, Tendoro KV, Dalto LG, Pacanaro SV, Lodi CS, de Melo FFF (2010) Tissue reaction to silver nanoparticles dispersion as an alternative irrigating solution. J Endod 36: 1698-1702.

Gomes-Filho JE, Sivieri-Araujo G, Sipert CR, da Silva Santos LM, de Azevedo Queiroz ÍO, Men Martins C, do Carmo Maia NK, Cintra LTA, DezanJunior E, Bagnato VS, Chaves-Neto AH, de Oliveira SHP (2016) Evaluation of photodynamic therapy on fibroblast viability and cytokine production. Photodiagn Photodyn Ther 13: 97-100.

Grange JM, Davey RW (1990) Antibacterial properties of propolis (bee glue). J R Soc Med 83: 159-160.

Grant W, MacRobert A, Bown S, Hopper C, Speight P (1993) Photodynamic therapy of oral cancer: photosensitisation with systemic aminolaevulinic acid. Lancet 342: 147-148.

Guazzo R, Gardin C, Bellin G, Sbricoli L, Ferroni L, Ludovichetti FS, Piattelli A, Antoniac I, Bressan E, Zavan B (2018) Graphene-based nanomaterials for tissue engineering in the dental field. J Nanomater 8: 349. DOI: 10.3390/nano8050349.

Gubler M, Brunner TJ, Zehnder M, Waltimo T, Sener B, Stark WJ (2008) Do bioactive glasses convey a disinfecting mechanism beyond a mere increase in pH? Int Endod J 41: 670-678.

Gursoy H, Ozcakir-Tomruk C, Tanalp J, Yilmaz S (2013) Photodynamic therapy in dentistry: a literature review. Clin Oral Investig 17: 1113-1125.

Gurzawska K, Dirscherl K, Jørgensen B, Berglundh T, Jørgensen NR, Gotfredsen K (2017) Pectin nanocoating of titanium implant surfaces - an experimental study in rabbits. Clin Oral Implants Res 28: 298-307.

Halboub E, Al-Maweri SA, Al-Wesabi M, AlKamel A, Shamala A, Al-Sharani A, Koppolu P (2020) Efficacy of propolis-based mouthwashes on dental plaque and gingival inflammation: a systematic review. BMC Oral Health 20: 198-198.

Hamblin MR, Hasan T (2004) Photodynamic therapy: a new antimicrobial approach to infectious disease? Photochem Photobiol Sci 3: 436-450.

Hench LL, Paschall H (1973) Direct chemical bond of bioactive glass-ceramic materials to bone and muscle. J Biomed Mater Res 7: 25-42.

Hench LL, Splinter RJ, Allen W, Greenlee T (1971) Bonding mechanisms at the interface of ceramic prosthetic materials. J Biomed Mater Res 5: 117-141. 
Hewlings SJ, Kalman DS (2017) Curcumin: a review of its effects on human health. Foods 6: 92.

Hopper C (2000) Photodynamic therapy: a clinical reality in the treatment of cancer. Lancet Oncol 1: 212-219.

Ioannidis K, Niazi S, Mylonas P, Mannocci F, Deb S (2019) The synthesis of nano silver-graphene oxide system and its efficacy against endodontic biofilms using a novel tooth model. Dent Mater 35: 1614-1629.

Ishii T, Matsunaga T (2001) Pectic polysaccharide rhamnogalacturonan II is covalently linked to homogalacturonan. Phytochemistry 57: 969-974.

Iwaya S-i, Ikawa M, Kubota M (2001) Revascularization of an immature permanent tooth with apical periodontitis and sinus tract. Dent Traumatol 17: 185-187.

Johns DA, Shivashankar VY, Krishnamma S, Johns M (2014) Use of photoactivated disinfection and platelet-rich fibrin in regenerative endodontics. J Conserv Dent 17: 487-490.

Jung HS, Lee T, Kwon IK, Kim HS, Hahn SK, Lee CS (2015) Surface modification of multipass caliberrolled ti alloy with dexamethasone-loaded graphene for dental applications. ACS Appl Mater Interfaces 7: 9598-9607.

Khalil ML (2006) Biological activity of bee propolis in health and disease. Asian Pac J Cancer Prev 7: 2231.

Kim J-H, Kim Y, Shin S-J, Park J-W, Jung I-Y (2010) Tooth discoloration of immature permanent incisor associated with triple antibiotic therapy: a case report. J Endod 36: 1086-1091.

Kim SG, Malek M, Sigurdsson A, Lin LM, Kahler B (2018) Regenerative endodontics: a comprehensive review. Int Endod J 51: 1367-1388.

Konopka K, Goslinski T (2007) Photodynamic therapy in dentistry. J Dent Res 86: 694-707.

Koo H, Gomes BPFA, Rosalen PL, Ambrosano GMB, Park YK, Cury JA (2000) In vitro antimicrobial activity of propolis and Arnica montana against oral pathogens. Arch Oral Biol 45: 141-148.

Koo Y-EL, Fan W, Hah H, Xu H, Orringer D, Ross B, Rehemtulla A, Philbert MA, Kopelman R (2007) Photonic explorers based on multifunctional nanoplatforms for biosensing and photodynamic therapy. Appl Opt 46: 1924-1930.

Krithikadatta J, Indira R, Dorothykalyani AL (2007) Disinfection of dentinal tubules with $2 \%$ chlorhexidine, $2 \%$ metronidazole, bioactive glass when compared with calcium hydroxide as intracanal medicaments. J Endod 33: 1473-1476.

Lara HH, Ayala-Núñez NV, Ixtepan Turrent LdC, Rodríguez Padilla C (2010) Bactericidal effect of silver nanoparticles against multidrug-resistant bacteria. World J Microbiol Biotechnol 26: 615-621.

Lei B, Chen X, Wang Y, Zhao N, Du C, Fang L (2010) Surface nanoscale patterning of bioactive glass to support cellular growth and differentiation. J Biomed Mater Res A 94A: 1091-1099.

Lenherr P, Allgayer N, Weiger R, Filippi A, Attin T, Krastl G (2012) Tooth discoloration induced by endodontic materials: a laboratory study. Int Endod J 45: 942-949.

Li J, Cai C, Li J, Li J, Li J, Sun T, Wang L, Wu H, Yu G (2018) Chitosan-based nanomaterials for drug delivery. Molecules 23: 2661. DOI: 10.3390/ molecules23102661.

Li J, Wang G, Zhu H, Zhang M, Zheng X, Di Z, Liu X, Wang X (2014) Antibacterial activity of large-area monolayer graphene film manipulated by charge transfer. Sci Rep 4: 4359. DOI: 10.1038/srep04359.

Li Y, Ge H, Wu L, Lei L, Wang Y, Jiang S, Cai Z, Huang X (2020) Pretreatment of root canal with photodynamic therapy facilitates adhesion, viability, and differentiation of stem cells of the apical papilla. Photochem Photobiol 96: 890-896.

Liu S, Zeng TH, Hofmann M, Burcombe E, Wei J, Jiang R, Kong J, Chen Y (2011) Antibacterial activity of graphite, graphite oxide, graphene oxide, and reduced graphene oxide: membrane and oxidative stress. ACS Nano 5: 6971-6980.

Ma J, Tong Z, Ling J, Liu H, Wei X (2015) The effects of sodium hypochlorite and chlorhexidine irrigants on the antibacterial activities of alkaline media against Enterococcus faecalis. Arch Oral Biol 60: 1075-1081.

Marcucci M (1995) Propolis: chemical composition, biological properties and therapeutic activity. Apidologie 26: 83-99.

Martin DE, De Almeida JFA, Henry MA, Khaing ZZ, Schmidt CE, Teixeira FB, Diogenes A (2014) Concentration-dependent effect of sodium hypochlorite on stem cells of apical papilla survival and differentiation. J Endod 40: 51-55.

Martin MP, Pileggi R (2004) A quantitative analysis of propolis: a promising new storage media following avulsion. Dent Traumatol 20: 85-89.

Men'shikov DD, Lazareva EB, Popova TS, Shramko LU, Tokaev IS, Zalogueva GV, Gaponova IN (1997) Antimicrobial properties of pectins and their effects on antibiotics. Antibiot Chemother 42: 10-15.

Menaa F, Abdelghani A, Menaa B (2015) Graphene nanomaterials as biocompatible and conductive scaffolds for stem cells: impact for tissue engineering and regenerative medicine. J Tissue Eng Regen Med 9: 1321-1338.

Mishra RK, Datt M, Pal K, Banthia A (2008) Preparation and characterization of amidated pectin based hydrogels for drug delivery system. J Mater Sci Mater Med 19: 2275-2280.

Moazami F, Sahebi S, Ahzan S (2018) Tooth discoloration induced by imidazolium based silver nanoparticles as an intracanal irrigant. J Dent (Shiraz) 19: 280-286.

Montero JC, Mori GG (2012) Assessment of ion diffusion from a calcium hydroxide-propolis paste through dentin. Braz Oral Res 26: 318-322.

Morawiec T, Dziedzic A, Niedzielska I, Mertas A, Tanasiewicz M, Skaba D, Kasperski J, MachorowskaPieniążek A, Kucharzewski M, Szaniawska K, Więckiewicz W, Więckiewicz M (2013) The biological activity of propolis-containing toothpaste on oral 
health environment in patients who underwent implant-supported prosthodontic rehabilitation. Evid Based Complement Alternat Med 2013: 704947 704947.

Morris MD, Lee K-W, Agee KA, Bouillaguet S, Pashley DH (2001) Effects of sodium hypochlorite and rc-prep on bond strengths of resin cement to endodontic Surfaces. J Endod 27: 753-757.

Munarin F, Giuliano L, Bozzini S, Tanzi MC, Petrini P (2010a) Mineral phase deposition on pectin microspheres. Mater Sci Eng C 30: 491-496.

Munarin F, Guerreiro SG, Grellier MA, Tanzi MC, Barbosa MA, Petrini P, Granja PL (2011) Pectin-based injectable biomaterials for bone tissue engineering. Biomacromolecules 12: 568-577.

Munarin F, Petrini P, Farè S, Tanzi MC (2010b) Structural properties of polysaccharide-based microcapsules for soft tissue regeneration. J Mater Sci Mater Med 21: 365-375.

Munarin F, Petrini P, Tanzi MC, Barbosa MA, Granja PL (2012) Biofunctional chemically modified pectin for cell delivery. Soft Matter 8: 4731-4739.

Munukka E, Leppäranta O, Korkeamäki M, Vaahtio M, Peltola T, Zhang D, Hupa L, Ylänen H, Salonen JI, Viljanen MK, Eerola E (2008) Bactericidal effects of bioactive glasses on clinically important aerobic bacteria. J Mater Sci Mater Med 19: 27-32.

Murray PE, Garcia-Godoy F, Hargreaves KM (2007) Regenerative endodontics: a review of current status and a call for action. J Endod 33: 377-390.

Nazzal H, Kenny K, Altimimi A, Kang J, Duggal MS (2018) A prospective clinical study of regenerative endodontic treatment of traumatized immature teeth with necrotic pulps using bi-antibiotic paste. Int Endod J 51: e204-e215.

Neelakantan P, Cheng CQ, Ravichandran V, Mao T, Sriraman P, Sridharan S, Subbarao C, Sharma S, Kishen A (2015) Photoactivation of curcumin and sodium hypochlorite to enhance antibiofilm efficacy in root canal dentin. Photodiagn Photodyn Ther 12: 108-114.

Neelakantan P, Subbarao C, Sharma S, Subbarao CV, Garcia-Godoy F, Gutmann JL (2013) Effectiveness of curcumin against Enterococcus faecalis biofilm. Acta Odontol Scand 71: 1453-1457.

Newby PJ, El-Gendy R, Kirkham J, Yang XB, Thompson ID, Boccaccini AR (2011) Ag-doped 45S5 Bioglass -based bone scaffolds by molten salt ion exchange: processing and characterisation. J Mater Sci Mater Med 22: 557-569.

Nguyen S, Hiorth M, Rykke M, Smistad G (2013) Polymer coated liposomes for dental drug delivery - interactions with parotid saliva and dental enamel. Eur J Pharm Sci 50: 78-85.

Noronha VT, Paula AJ, Durán G, Galembeck A, Cogo-Müller K, Franz-Montan M, Durán N (2017) Silver nanoparticles in dentistry. Dent Mater 33: 1110-1126.

Norton MR, Wilson J (2002) Dental implants placed in extraction sites implanted with bioactive glass: human histology and clinical outcome. Int J Oral Maxillofac Implants 17: 249-257.

Novoselov KS, Geim AK, Morozov SV, Jiang D, Zhang Y, Dubonos SV, Grigorieva IV, Firsov AA (2004) Electric field effect in atomically thin carbon films. Science 306: 666. DOI: 10.1126/science.1102896.

Nunes MR, Mello I, Franco GC, de Medeiros JM, Dos Santos SS, Habitante SM, Lage-Marques JL, Raldi DP (2011) Effectiveness of photodynamic therapy against Enterococcus faecalis, with and without the use of an intracanal optical fiber: an in vitro study. Photomed Laser Surg 29: 803-808.

Oei JD, Zhao WW, Chu L, DeSilva MN, Ghimire A, Rawls HR, Whang K (2012) Antimicrobial acrylic materials with in situ generated silver nanoparticles. J Biomed Mater Res B Appl Biomate 100B: 409-415.

Okino LA, Siqueira EL, Santos M, Bombana AC, Figueiredo JAP (2004) Dissolution of pulp tissue by aqueous solution of chlorhexidine digluconate and chlorhexidine digluconate gel. Int Endod J 37: 38-41.

Otoni CG, Moura MRd, Aouada FA, Camilloto GP, Cruz RS, Lorevice MV, Soares NdFF, Mattoso LHC (2014) Antimicrobial and physical-mechanical properties of pectin/papaya puree/cinnamaldehyde nanoemulsion edible composite films. Food Hydrocoll 41: 188-194.

Ozan F, Polat ZA, Er K, Ozan U, Değer O (2007) Effect of propolis on survival of periodontal ligament cells: new storage media for avulsed teeth. J Endod 33: 570-573.

Pagliarin CML, Londero CDLD, Felippe MCS, Felippe WT, Danesi CC, Barletta FB (2016) Tissue characterization following revascularization of immature dog teeth using different disinfection pastes. Braz Oral Res 30: S1806. DOI: 10.1590/18073107BOR-2016.vol30.0074.

Pagonis TC, Chen J, Fontana CR, Devalapally H, Ruggiero K, Song X, Foschi F, Dunham J, Skobe Z, Yamazaki H (2010) Nanoparticle-based endodontic antimicrobial photodynamic therapy. J Endod 36: 322-328.

Palma PJ, Ramos JC, Martins JB, Diogenes A, Figueiredo MH, Ferreira P, Viegas C, Santos JM (2017) Histologic evaluation of regenerative endodontic procedures with the use of chitosan scaffolds in immature dog teeth with apical periodontitis. J Endod 43: $1279-1287$.

Pascon FM, Kantovitz KR, Sacramento PA, Nobre-dos-Santos M, Puppin-Rontani RM (2009) Effect of sodium hypochlorite on dentine mechanical properties. a review. J Dent 37: 903-908.

Pereira RS, Menezes JD, Bonardi JP, Griza GL, Okamoto R, Hochuli-Vieira E (2018) Comparative study of volumetric changes and trabecular microarchitecture in human maxillary sinus bone augmentation with bioactive glass and autogenous bone graft: a prospective and randomized assessment. Int J Oral Maxillofac Surg 47: 665-671.

Peter MG (1995) Applications and environmental aspects of chitin and chitosan. J Macromol Sci A 32: 629-640. 
Plotino G, Grande NM, Mercade M (2019) Photodynamic therapy in endodontics. Int Endod J 52: 760-774.

Pourhajibagher M, Chiniforush N, Shahabi S, Palizvani M, Bahador A (2018) Antibacterial and antibiofilm efficacy of antimicrobial photodynamic therapy against intracanal Enterococcus faecalis: an in vitro comparative study with traditional endodontic irrigation solutions. J Dent (Tehran) 15: 197-204.

Praditya D, Kirchhoff L, Brüning J, Rachmawati H, Steinmann J, Steinmann E (2019) Anti-infective properties of the golden spice curcumin. Front Microbiol 10: 912. DOI: 10.3389/fmicb.2019.00912.

Raafat D, Sahl H-G (2009) Chitosan and its antimicrobial potential - a critical literature survey. Microb Biotechnol 2: 186-201.

Rabea EI, Badawy MET, Stevens CV, Smagghe G, Steurbaut W (2003) Chitosan as antimicrobial agent: applications and mode of action. Biomacromolecules 4: 1457-1465.

Abdel Hafiz Abdel Rahim A-S, Abdelgawad F, Abd Alsamed AM, Moheb DM, Wahab El-Dokky NA (2019) Case report: single visit photo-activated disinfection in regenerative endodontics. F1000Res 8: 1519. DOI: 10.12688/f1000research.20118.2.

Rai D, Singh Jay K, Roy N, Panda D (2008) Curcumin inhibits FtsZ assembly: an attractive mechanism for its antibacterial activity. Biochem J 410: 147-155.

Rai M, Ingle AP, Pandit R, Paralikar P, Anasane N, Santos CAD (2020) Curcumin and curcumin-loaded nanoparticles: antipathogenic and antiparasitic activities. Expert Rev Anti Infect Ther 18: 367-379.

Rai M, Yadav A, Gade A (2009) Silver nanoparticles as a new generation of antimicrobials. Biotechnol Adv 27: 76-83.

Ramalho KM, Cunha SR, Mayer-Santos E, Eduardo CdP, Freitas PMd, Aranha ACC, MouraNetto C (2017) In vitro evaluation of methylene blue removal from root canal after photodynamic therapy. Photodiagn Photodyn Ther 20: 248-252.

Redondo-Nevado J, Moyano E, MedinaEscobar N, Caballero JL, Muñoz-Blanco J (2001) A fruit-specific and developmentally regulated endopolygalacturonase gene from strawberry (Fragaria x ananassa cv. Chandler). J Exp Bot 52: 19411945.

Ren N, Li J, Qiu J, Yan M, Liu H, Ji D, Huang J, Yu J, Liu H (2017) Growth and accelerated differentiation of mesenchymal stem cells on graphene-oxide-coated titanate with dexamethasone on surface of titanium implants. Dent Mater 33: 525-535.

Renard E, Amiaud J, Delbos L, Charrier C, Montembault A, Ducret M, Farges F, David L, AlliotLicht B, Gaudin A (2020) Dental pulp inflammatory/ immune response to a chitosan-enriched fibrin hydrogel in the pulpotomised rat incisor. Eur Cell Mater 40: 74-87.

de Rezende GP, da Costa LR, Pimenta FC, Baroni DA (2008) In vitro antimicrobial activity of endodontic pastes with propolis extracts and calcium hydroxide: a preliminary study. Braz Dent J 19: 301-305.

Ribeiro JS, Münchow EA, Ferreira Bordini EA, de Oliveira da Rosa WL, Bottino MC (2020) Antimicrobial therapeutics in regenerative endodontics: a scoping review. J Endod 46: S115-S127.

Ring KC, Murray PE, Namerow KN, Kuttler S, Garcia-Godoy F (2008) The comparison of the effect of endodontic irrigation on cell adherence to root canal dentin. J Endod 34: 1474-1479.

Rosa V, Xie H, Dubey N, Madanagopal TT, Rajan SS, Morin JLP, Islam I, Castro Neto AH (2016) Graphene oxide-based substrate: physical and surface characterization, cytocompatibility and differentiation potential of dental pulp stem cells. Dent Mater 32: 1019-1025.

Ruparel NB, Teixeira FB, Ferraz CC, Diogenes A (2012) Direct effect of intracanal medicaments on survival of stem cells of the apical papilla. J Endod 38: 1372-1375.

Sadek RW, Moussa SM, El Backly RM, Hammouda AF (2019) Evaluation of the efficacy of three antimicrobial agents used for regenerative endodontics: an in vitro study. Microb Drug Resist 25: 761-771.

Samiei M, Aghazadeh M, Lotfi M, Shakoei S, Aghazadeh Z, Vahid Pakdel SM (2013) Antimicrobial efficacy of mineral trioxide aggregate with and without silver nanoparticles. Iran Endod J 8: 166-170.

Samiei M, Farjami A, Dizaj SM, Lotfipour F (2016) Nanoparticles for antimicrobial purposes in endodontics: a systematic review of in vitro studies. Mater Sci Eng C 58: 1269-1278.

Sato I, Ando-Kurihara N, Kota K, Iwaku M, Hoshino E (1996) Sterilization of infected rootcanal dentine by topical application of a mixture of ciprofloxacin, metronidazole and minocycline in situ. Int Endod J 29: 118-124.

Sauro S, Osorio R, Watson TF, Toledano M (2012) Therapeutic effects of novel resin bonding systems containing bioactive glasses on mineral-depleted areas within the bonded-dentine interface. J Mater Sci Mater Med 23: 1521-1532.

Sforcin JM, Bankova V (2011) Propolis: is there a potential for the development of new drugs? J Ethnopharmacol 133: 253-260.

Shahravan A, Haghdoost A-A, Adl A, Rahimi H, Shadifar F (2007) Effect of smear layer on sealing ability of canal obturation: a systematic review and meta-analysis. J Endod 33: 96-105.

Shaik J, Garlapati R, Nagesh B, Sujana V, Jayaprakash T, Naidu S (2014) Comparative evaluation of antimicrobial efficacy of triple antibiotic paste and calcium hydroxide using chitosan as carrier against Candida albicans and Enterococcus faecalis: an in vitro study. J Conserv Dent 17: 335-339.

Sheng X-Y, Gong W-Y, Hu Q, Chen X-f, Dong Y-M (2016) Mineral formation on dentin induced by nano-bioactive glass. Chin Chem Lett 27: 1509-1514.

Shrestha A, Cordova M, Kishen A (2015) Photoactivated polycationic bioactive chitosan 
nanoparticles inactivate bacterial endotoxins. J Endod 41: 686-691.

Shrestha A, Kishen A (2014) Antibacterial efficacy of photosensitizer functionalized biopolymeric nanoparticles in the presence of tissue inhibitors in root canal. J Endod 40: 566-570.

Shrestha A, Shi Z, Neoh KG, Kishen A (2010) Nanoparticulates for antibiofilm treatment and effect of aging on its antibacterial activity. J Endod 36: 1030 1035.

Siddiqui SH, Awan KH, Javed F (2013) Bactericidal efficacy of photodynamic therapy against Enterococcus faecalis in infected root canals: a systematic literature review. Photodiagn Photodyn Ther 10: 632-643.

Sinjari B, Pizzicannella J, D’ Aurora M, Zappacosta R, Gatta V, Fontana A, Trubiani O, Diomede F (2019) Curcumin/liposome nanotechnology as delivery platform for anti-inflammatory activities via NFkB/ ERK/pERK pathway in human dental pulp treated with 2-hydroxyethyl methacrylate (HEMA). Front Physiol 10. DOI: 10.3389/fphys.2019.00633.

Smirnoff N (2000) Ascorbic acid: metabolism and functions of a multi-facetted molecule. Curr Opin Plant Biol 3: 229-235.

Sotomil JM, Münchow EA, Pankajakshan D, Spolnik KJ, Ferreira JA, Gregory RL, Bottino MC (2019) Curcumin-a natural medicament for root canal disinfection: effects of irrigation, drug release, and photoactivation. J Endod 45: 1371-1377.

Soukos NS, Chen PS-Y, Morris JT, Ruggiero K, Abernethy AD, Som S, Foschi F, Doucette S, Luschke Bammann L, Fontana CR, Doukas AG, Stashenko PP (2006) Photodynamic therapy for endodontic disinfection. J Endod 32: 979-984.

Stewart PS, William Costerton J (2001) Antibiotic resistance of bacteria in biofilms. Lancet 358: 135-138.

Sun L, Yan Z, Duan Y, Zhang J, Liu B (2018) Improvement of the mechanical, tribological and antibacterial properties of glass ionomer cements by fluorinated graphene. Dent Mater 34: e115-e127.

Sut S, Baldan V, Faggian M, Peron G, Dall 'Acqua S (2016) Nutraceuticals, a new challenge for medicinal chemistry. Curr Med Chem 23: 3198-3223.

Tagelsir A, Yassen GH, Gomez GF, Gregory RL (2016) Effect of antimicrobials used in regenerative endodontic procedures on 3-week-old Enterococcus faecalis biofilm. J Endod 42: 258-262.

Tahmassebi JF, Drogkari E, Wood SR (2015) A study of the control of oral plaque biofilms via antibacterial photodynamic therapy. Eur Arch Paediatr Dent 16: 433-440.

Tahriri M, Del Monico M, Moghanian A, Tavakkoli Yaraki M, Torres R, Yadegari A, Tayebi L (2019) Graphene and its derivatives: opportunities and challenges in dentistry. Mater Sci Eng C 102: 171-185.

Tirapelli C, Panzeri H, Lara EH, Soares RG, Peitl O, Zanotto ED (2011) The effect of a novel crystallised bioactive glass-ceramic powder on dentine hypersensitivity: a long-term clinical study. J Oral Rehabil 38: 253-262.
Tong HJ, Rajan S, Bhujel N, Kang J, Duggal M, Nazzal H (2017) Regenerative endodontic therapy in the management of nonvital immature permanent teeth: a systematic review - outcome evaluation and meta-analysis. J Endod 43: 1453-1464.

Tong HJ, Sim YF, Berdouses E, Al-Jundi S, El Shahawy O, Nazzal H (2020) Regenerative endodontic therapy (RET) for managing immature non-vital teeth: experiences and opinions of paediatric dental practitioners in the European and Arabian regions. Eur Arch Paediatr Dent: 1-11.

Trevino EG, Patwardhan AN, Henry MA, Perry G, Dybdal-Hargreaves N, Hargreaves KM, Diogenes A (2011) Effect of irrigants on the survival of human stem cells of the apical papilla in a platelet-rich plasma scaffold in human root tips. J Endod 37: 11091115.

Tyagi P, Singh M, Kumari H, Kumari A, Mukhopadhyay K (2015) Bactericidal activity of curcumin $\mathrm{i}$ is associated with damaging of bacterial membrane. PLoS One 10: e0121313. DOI: 10.1371/ journal.pone.0121313.

Uzel A, Sorkun Ky, Önçağ Ö, Çoğulu D, Gençay Ö, Sali h Br (2005) Chemical compositions and antimicrobial activities of four different anatolian propolis samples. Microbiol Res 160: 189-195.

Varoni EM, Iriti M, Rimondini L (2012) Plant products for innovative biomaterials in dentistry. Coatings 2: 179-194.

Vilela Teixeira AB, Vidal CL, de Castro DT, da Costa Valente ML, Oliveira-Santos C, Alves OL, Dos Reis AC (2017) Effect of incorporation of a new antimicrobial nanomaterial on the physical-chemical properties of endodontic sealers. J Conserv Dent 20: 392-397.

Vincken J-P, Schols HA, Oomen RJFJ, McCann MC, Ulvskov P, Voragen AGJ, Visser RGF (2003) If homogalacturonan were a side chain of rhamnogalacturonan i. implications for cell wall architecture. Plant Physiol 132: 1781-1789.

Wainwright M, Crossley KB (2004) Photosensitising agents - circumventing resistance and breaking down biofilms: a review. Int Biodeterior Biodegradation 53: 119-126.

Waltimo T, Brunner TJ, Vollenweider M, Stark WJ, Zehnder M (2007) Antimicrobial effect of nanometric bioactive glass 45C5. J Dent Res 86: 754-757.

Wang S, Huang G, Dong Y (2020) Directional migration and odontogenic differentiation of bone marrow stem cells induced by dentin coated with nanobioactive glass. J Endod 46: 216-223.

Wang Y, Chatzistavrou X, Faulk D, Badylak S, Zheng L, Papagerakis S, Ge L, Liu H, Papagerakis P (2015) Biological and bactericidal properties of Agdoped bioactive glass in a natural extracellular matrix hydrogel with potential application in dentistry. Eur Cell Mater 29: 342-355.

Wang Z, Sun Y, Wang D, Liu H, Boughton RI (2013) In situ fabrication of silver nanoparticlefilled hydrogen titanate nanotube layer on metallic 
titanium surface for bacteriostatic and biocompatible implantation. Int J Nanomedicine 8: 2903-2916.

Widbiller M, Althumairy RI, Diogenes A (2019) Direct and indirect effect of chlorhexidine on survival of stem cells from the apical papilla and its neutralization. J Endod 45: 156-160.

Wood S, Metcalf D, Devine D, Robinson C (2006) Erythrosine is a potential photosensitizer for the photodynamic therapy of oral plaque biofilms. J Antimicrob Chemother 57: 680-684.

Wu C, Chang J (2014) Multifunctional mesoporous bioactive glasses for effective delivery of therapeutic ions and drug/growth factors. J Control Release 193: 282-295.

Wu C, Chang J, Xiao Y (2011) Mesoporous bioactive glasses as drug delivery and bone tissue regeneration platforms. Ther Deliv 2: 1189-1198.

Wu D, Fan W, Kishen A, Gutmann JL, Fan B (2014) Evaluation of the antibacterial efficacy of silver nanoparticles against Enterococcus faecalis biofilm. J Endod 40: 285-290.

Xie H, Chua M, Islam I, Bentini R, Cao T, VianaGomes JC, Castro Neto AH, Rosa V (2017) CVDgrown monolayer graphene induces osteogenic but not odontoblastic differentiation of dental pulp stem cells. Dent Mater 33: e13-e21.

Xuereb M, Camilleri J, Attard NJ (2015) Systematic review of current dental implant coating materials and novel coating techniques. Int J Prosthodont 28: 51-59.

Yadav R, Tikku A, Chandra A, Verma P, Bains R, Bhoot H (2018) A comparative evaluation of the antimicrobial efficacy of calcium hydroxide, chlorhexidine gel, and a curcumin-based formulation against Enterococcus faecalis. Natl J Maxillofac Surg 9: 52-55.

Yadlapati M, Biguetti C, Cavalla F, Nieves F, Bessey C, Bohluli P, Garlet GP, Letra A, Fakhouri WD, Silva RM (2017) Characterization of a vascular endothelial growth factor-loaded bioresorbable delivery system for pulp regeneration. J Endod 43: 77-83.

Yadlapati M, Souza LC, Dorn S, Garlet GP, Letra A, Silva RM (2014) Deleterious effect of triple antibiotic paste on human periodontal ligament fibroblasts. Int Endod J 47: 769-775.

Yamada S, Yamamoto K, Ikeda T, Yanagiguchi K, Hayashi Y (2014) Potency of fish collagen as a scaffold for regenerative medicine. Biomed Res Int 2014: 302932. DOI: 10.1155/2014/302932.

Yan X, Yu C, Zhou X, Tang J, Zhao D (2004) Highly ordered mesoporous bioactive glasses with superior in vitro bone-forming bioactivities. Angew Chem Int Ed 43: 5980-5984.
Yassen GH, Chu T-MG, Eckert G, Platt JA (2013a) Effect of medicaments used in endodontic regeneration technique on the chemical structure of human immature radicular dentin: an in vitro study. J Endod 39: 269-273.

Yassen GH, Vail MM, Chu TG, Platt JA (2013b) The effect of medicaments used in endodontic regeneration on root fracture and microhardness of radicular dentine. Int Endod J 46: 688-695.

Zehnder M (2006) Root canal irrigants. J Endod 32: 389-398.

Zehnder M, Luder HU, Schätzle M, Kerosuo E, Waltimo T (2006) A comparative study on the disinfection potentials of bioactive glass S53P4 and calcium hydroxide in contra-lateral human premolars ex vivo. Int Endod J 39: 952-958.

Zehnder M, Söderling E, Salonen J, Waltimo T (2004) Preliminary evaluation of bioactive glass S53P4 as an endodontic medication in vitro. J Endod 30: 220224.

Zeng Q, Nguyen S, Zhang H, Chebrolu HP, Alzebdeh D, Badi MA, Kim JR, Ling J, Yang M (2016) Release of growth factors into root canal by irrigations in regenerative endodontics. J Endod 42: 1760-1766.

Zhang D, Leppäranta O, Munukka E, Ylänen H, Viljanen MK, Eerola E, Hupa M, Hupa L (2010) Antibacterial effects and dissolution behavior of six bioactive glasses. J Biomed Mater Res A 93A: 475-483.

Zhou R, Gao H (2014) Cytotoxicity of graphene: recent advances and future perspective. Wiley Interdiscip Rev Nanomed Nanobiotechnol 6: 452-474.

Zhu J, Wang J, Hou J, Zhang Y, Liu J, Van der Bruggen B (2017) Graphene-based antimicrobial polymeric membranes: a review. J Mater Chem A 5: 6776-6793.

Zhu N, Chatzistavrou X, Ge L, Qin M, Papagerakis P, Wang Y (2019) Biological properties of modified bioactive glass on dental pulp cells. J Dent 83: 18-26.

\section{Web reference}

1. AAE (2018) American Association of Endodontists, Clinical considerations for a regenerative procedure (Revised). https:// f3f142zs0k2w1kg84k5p9i1o-wpengine.netdna-ssl. com/specialty/wp-content/uploads/sites/2/2018/06/ ConsiderationsForRegEndo_AsOfApril2018.pdf [0906-2021].

Editor's note: There were no questions from reviewers for this paper, therefore there is no Discussion with Reviewers section. The Scientific Editor responsible for this paper was Thimios Mitsiadis. 